\title{
Classification of the Quasifiliform Nilpotent Lie Algebras of Dimension 9
}

\author{
Mercedes Pérez, ${ }^{1}$ Francisco P. Pérez, ${ }^{2}$ and Emilio Jiménez ${ }^{3}$ \\ ${ }^{1}$ Department of Mechanical Engineering, University of La Rioja, 26004 Logroño, Spain \\ ${ }^{2}$ Department of Applied Mathematics I, University of Sevilla, 41012 Seville, Spain \\ ${ }^{3}$ Department of Electrical Engineering, University of La Rioja, 26004 Logroño, Spain
}

Correspondence should be addressed to Emilio Jiménez; emilio.jimenez@unirioja.es

Received 3 November 2013; Accepted 2 January 2014; Published 6 March 2014

Academic Editor: Peter G. L. Leach

Copyright (c) 2014 Mercedes Pérez et al. This is an open access article distributed under the Creative Commons Attribution License, which permits unrestricted use, distribution, and reproduction in any medium, provided the original work is properly cited.

\begin{abstract}
On the basis of the family of quasifiliform Lie algebra laws of dimension 9 of 16 parameters and 17 constraints, this paper is devoted to identify the invariants that completely classify the algebras over the complex numbers except for isomorphism. It is proved that the nullification of certain parameters or of parameter expressions divides the family into subfamilies such that any couple of them is nonisomorphic and any quasifiliform Lie algebra of dimension 9 is isomorphic to one of them. The iterative and exhaustive computation with Maple provides the classification, which divides the original family into 263 subfamilies, composed of 157 simple algebras, 77 families depending on 1 parameter, 24 families depending on 2 parameters, and 5 families depending on 3 parameters.
\end{abstract}

\section{Introduction}

The interest in classifying nilpotent Lie algebras is broad both within the academic community and the industrial engineering community, since they are applied in classical mechanical problems and current research in scientific disciplines as modern geometry, solid state physics, or particle physics [15]. Lie algebras classification consists in determining equivalence relations that subdivide the original set in equivalence classes defined by at least one element in each set, and it is usual to classify the algebras except for isomorphisms. The solvable Lie algebras classification problem comes down in a sense to the nilpotent Lie algebras classification [6] and computer algebra has been indispensable. However, the more the dimension increases, the more and more complex is the determination of exhaustive lists of Lie algebras, so new computation methodologies are a present field of research $[7,8]$ with current symbolic manipulation programs such as Reduce, Mathematica, or Maple [9].

The classification of nilpotent Lie algebras over the complex numbers experimented an important advance based on the works of Ancochéa-Bermúdez and Goze [10] introducing an invariant more potent than the previously existing: the characteristic sequence or Goze's invariant (defined in
Section 2.1). Those authors were able, by using the characteristic sequence as an invariant, to classify the nilpotent Lie algebras of dimension 7 [11] and the filiform Lie algebras of dimension 8 [12]. Later, by using that invariant, Gomez and Echarte [13] classify the filiform Lie algebras of dimension 9. Afterward, Castro et al. [14] develop an algorithm for symbolic language for finding the generic families of filiform Lie algebras in any dimension with the restrictions required to the parameters.

Subsequent works about quasifiliform Lie algebras classification were centered on specific types of families or subclasses, obtaining results applicable to higher dimensions. For instance, the classifications of naturally graded [15] and graded by derivations [16] quasifiliform Lie algebras. These works extended to other algebras, with a high nilindex, the classification of graded filiform Lie algebras, studied initially by Vergne $[17,18]$, obtained from the gradation related to the filtration produced in a natural way by the descending central sequence.

In this paper we focus on a method of identification of the invariants that completely classify the nilpotent Lie algebras of dimension 9 over the complex numbers except for isomorphisms. With this aim, the dimensions of the subalgebras of its derived series, of its descending central 
series, and of its descending central series centralizers are used as class invariants. The exhaustive analysis has been developed with significant computational effort; the total code is 2820 pages in 37 files, summing more than 12000 lines of Maple code, and these programs have provided 3038 pages of results [19]. We strongly recommend the reading of Bäuerle and de Kerf [20], Benjumea et al. [21], and Sendra et al. [22] to become familiar with Lie algebras terminology and symbolic computation with Maple.

\section{The Subfamilies of Laws}

2.1. Preliminaries. Let $\mathfrak{g}$ be a nilpotent Lie algebra; the characteristic sequence of $a d(X)$ is denoted by $c(X)=\left(c_{1}, \ldots, c_{k}, 1\right)$, and for the lexicographic order $c(\mathfrak{g})=\operatorname{Max}_{X \in \mathfrak{g}-[\mathfrak{g}, \mathfrak{g}]} c(X)$ is known as the Goze's invariant or characteristic sequence [23]. Obviously $c(\mathfrak{g})$ is an invariant for the isomorphisms and, by construction, there is at least one vector $X \in \mathfrak{g}-[\mathfrak{g}, \mathfrak{g}]$ such that $c(\mathfrak{g})=c(X)$; all vector verifying this condition is called characteristic vector of the algebra.

The abelian algebra of dimension $n$ is the only one with Goze's invariant $(1, \ldots, 1)$, in metabelian algebras the characteristic series is $(2, \ldots 2,1, \ldots 1)$, in Heisenberg algebras it is $(2,1, \ldots, 1)$, in filiform algebras it is $(n-1,1)$, and in quasifiliform algebras it is $(n-2,1,1)$. A Lie algebra $\mathfrak{g}$ is nilpotent if and only if the characteristic polynomial of the matrix $a d(x)$ is $\lambda^{9}$, for every vector $x$ of $\mathfrak{g}$. Anyway this condition is often difficult to be applied, so the moment in the process, when the nilpotence condition should be applied or, much better, when the condition should be applied for each vector, has to be chosen carefully. The condition of being quasifiliform can be also interpreted in terms of matrices. Thus the vectors candidate to characteristic vectors, that is, the vectors in $\mathfrak{g}-[\mathfrak{g}, \mathfrak{g}]$, have to satisfy that the respective adjoint matrices do not have nonnull minors of order $\leqslant 7$. As in the case of the nilpotence, this condition has to be applied with caution and in several stages.

Every quasifiliform Lie algebra of dimension 9 can have an adapted base $\left\{x_{0}, x_{1}, \ldots, x_{8}\right\}$ such that

$$
\left[x_{0}, x_{i}\right]=x_{i+1}, \quad 1 \leqslant i \leqslant 6 ; \quad\left[x_{0}, x_{i}\right]=0, \quad 7 \leqslant i \leqslant 8 .
$$

On the whole all the bracket products can be described by

$$
\left[x_{i}, x_{j}\right]=\sum_{k=0}^{n-1} C_{i j}^{k} \cdot x_{k}, \quad 0 \leqslant i, j \leqslant n-1,
$$

where $C_{i j}^{k}$ are the algebra structure constants.

The laws of every complex quasifiliform Lie algebra (QFLA) of dimension 9 can be described by the following family with 16 parameters and 17 polynomial restriction equations [19] derived from the Jacobi identity:

$$
\begin{gathered}
{\left[x_{0}, x_{i}\right]=x_{i+1}, \quad 1 \leqslant i \leqslant 6,} \\
{\left[x_{1}, x_{2}\right]=\alpha_{1} x_{4}+\alpha_{2} x_{5}+\alpha_{3} x_{6}+\alpha_{4} x_{7}+\alpha_{5} x_{8},} \\
{\left[x_{1}, x_{3}\right]=\alpha_{1} x_{5}+\alpha_{2} x_{6}+\alpha_{3} x_{7}}
\end{gathered}
$$

$$
\begin{gathered}
{\left[x_{1}, x_{4}\right]=\alpha_{6} x_{5}+\alpha_{7} x_{6}+\alpha_{8} x_{7}+\alpha_{9} x_{8}} \\
{\left[x_{1}, x_{5}\right]=2 \alpha_{6} x_{6}+\left(2 \alpha_{7}-\alpha_{1}\right) x_{7}} \\
{\left[x_{1}, x_{6}\right]=\alpha_{10} x_{7}+\alpha_{11} x_{8}} \\
{\left[x_{1}, x_{8}\right]=\alpha_{12} x_{3}+\alpha_{13} x_{4}+\alpha_{14} x_{5}+\alpha_{15} x_{6}+\alpha_{16} x_{7}} \\
{\left[x_{2}, x_{3}\right]=-\alpha_{6} x_{5}+\left(\alpha_{1}-\alpha_{7}\right) x_{6}+\left(\alpha_{2}-\alpha_{8}\right) x_{7}-\alpha_{9} x_{8}} \\
{\left[x_{2}, x_{4}\right]=-\alpha_{6} x_{6}+\left(\alpha_{1}-\alpha_{7}\right) x_{7}} \\
{\left[x_{2}, x_{5}\right]=\left(2 \alpha_{6}-\alpha_{10}\right) x_{7}-\alpha_{11} x_{8}} \\
{\left[x_{2}, x_{8}\right]=\alpha_{12} x_{4}+\alpha_{13} x_{5}+\alpha_{14} x_{6}+\alpha_{15} x_{7}} \\
{\left[x_{3}, x_{4}\right]=\left(-3 \alpha_{6}+\alpha_{10}\right) x_{7}+\alpha_{11} x_{8}} \\
{\left[x_{3}, x_{8}\right]=\alpha_{12} x_{5}+\alpha_{13} x_{6}+\alpha_{14} x_{7}} \\
{\left[x_{4}, x_{8}\right]=\alpha_{12} x_{6}+\alpha_{13} x_{7}} \\
{\left[x_{5}, x_{8}\right]=\alpha_{12} x_{7}}
\end{gathered}
$$

subject to

$$
\begin{aligned}
\alpha_{5} \alpha_{12} & =0 \\
\alpha_{6} \alpha_{12} & =0 \\
\alpha_{6} \alpha_{13} & =0 \\
\alpha_{9} \alpha_{12} & =0 \\
\alpha_{9} \alpha_{13} & =0 \\
\alpha_{9} \alpha_{14} & =0 \\
\alpha_{10} \alpha_{12} & =0 \\
\alpha_{11} \alpha_{12} & =0 \\
\alpha_{11} \alpha_{13} & =0 \\
\alpha_{11} \alpha_{14} & =0 \\
\alpha_{11} \alpha_{15} & =0 \\
\alpha_{11} \alpha_{16} & =0 \\
\alpha_{5} \alpha_{14}-2\left(2 \alpha_{1}+\alpha_{7}\right) \alpha_{6}-\alpha_{9} \alpha_{16} \alpha_{12}+3\left(\alpha_{1}-\alpha_{7}\right) & =0 \\
\left.\alpha_{5} \alpha_{13}-2 \alpha_{6}-\alpha_{7}\right) & \left.\alpha_{13}+2\left(3 \alpha_{1}-\alpha_{7}\right) \alpha_{10}\right) \alpha_{14} \\
\left.\alpha_{1}-\alpha_{7}\right) & =0
\end{aligned}
$$

with the application of the Jacobi identity to the 3-tuple $\left(x_{0}, x_{i}, x_{j}\right)$, where $x_{i}, x_{j}$ are base vectors different from 
TABLE 1: Notation for the QFLA parameters.

\begin{tabular}{llll}
\hline$\alpha_{1}=C_{12}^{4}$ & $\alpha_{2}=C_{12}^{5}$ & $\alpha_{3}=C_{12}^{6}$ & $\alpha_{4}=C_{12}^{7}$ \\
$\alpha_{5}=C_{12}^{8}$ & $\alpha_{6}=C_{14}^{5}$ & $\alpha_{7}=C_{14}^{6}$ & $\alpha_{8}=C_{14}^{7}$ \\
$\alpha_{9}=C_{14}^{8}$ & $\alpha_{10}=C_{16}^{7}$ & $\alpha_{11}=C_{16}^{8}$ & $\alpha_{12}=C_{18}^{3}$ \\
$\alpha_{12}=C_{18}^{4}$ & $\alpha_{14}=C_{18}^{5}$ & $\alpha_{15}=C_{18}^{6}$ & $\alpha_{16}=C_{18}^{7}$ \\
\hline
\end{tabular}

$x_{0}$ vector. Table 1 shows the structure constants corresponding with the 16 parameters. From here forward the Lie Algebra Families will be denoted as $\mu\left(\alpha_{1}, \ldots, \alpha_{16}\right)$.

Our objective is to study exhaustively the case of dimension 9; therefore the coefficients identification is tackled in an iterative and interactive way by imposing the Jacobi identity. Maple programs have been developed so that all the equations resulting from the application of the abovementioned conditions are obtained, the simplest conditions are applied, and the process is repeated until there are no restrictions of simple application.

The exhaustiveness of the classification is developed by analyzing all the possible combinations of values of the 16 parameters $\left(\alpha_{1}, \ldots, \alpha_{16}\right)$, which is summarized within the cases shown in the following subsections: case A.1 $\left(\alpha_{11} \neq 0\right.$ and $\left.\alpha_{1} \neq 0\right)$, A.2 $\left(\alpha_{11} \neq 0\right.$ and $\left.\alpha_{1}=0\right)$, B.1.1 $\left(\alpha_{11}=0, \alpha_{9} \neq 0\right.$, and $\left.\alpha_{6} \neq 0\right)$, B.1.2 $\left(\alpha_{11}=0, \alpha_{9} \neq 0\right.$, and $\left.\alpha_{6}=0\right)$, B.2.1 $\left(\alpha_{11}=0\right.$, $\alpha_{9}=0$, and $\left.\alpha_{5} \neq 0\right)$, and B.2.2 $\left(\alpha_{11}=0, \alpha_{9}=0\right.$, and $\alpha_{5}=0$ ). In all the cases the nonisomorphism is proved in the corresponding propositions.

\subsection{General Case}

Proposition 1. The nilpotent $Q F L A$ of dimension 9 and $\alpha_{11} \neq 0$ are nonisomorphic to the algebras with $\alpha_{11}=0$.

Proof. For the family described by (3a)-(3o) and (4a)(4q), its descending central series is $\mathscr{C}^{1} \mathfrak{g}=\left\langle x_{2}, x_{3}, x_{4}\right.$, $\left.x_{5}, x_{6}, x_{7}, \alpha_{5} x_{8}, \alpha_{9} x_{8}, \alpha_{11} x_{8}\right\rangle, \mathscr{C}^{2} \mathfrak{g}=\left\langle x_{3}, x_{4}, x_{5}, x_{6}, x_{7}, \alpha_{5} x_{8}\right.$, $\left.\alpha_{9} x_{8}, \alpha_{11} x_{8}\right\rangle, \mathscr{C}^{3} \mathfrak{g}=\left\langle x_{4}, x_{5}, x_{6}, x_{7}, \alpha_{9} x_{8}, \alpha_{11} x_{8}\right\rangle, \mathscr{C}^{4} \mathfrak{g}=\left\langle x_{5}\right.$, $\left.x_{6}, x_{7}, \alpha_{9} x_{8}, \alpha_{11} x_{8}\right\rangle, \mathscr{C}^{5} \mathfrak{g}=\left\langle x_{6}, x_{7}, \alpha_{11} x_{8}\right\rangle$, and so forth. Thus $\operatorname{Dim}\left[\mathscr{C}^{5} \mathfrak{g}\right]=3$ if $\alpha_{11} \neq 0$ and $\operatorname{Dim}\left[\mathscr{C}^{5} \mathfrak{g}\right]=2$ if $\alpha_{11}=0$. Therefore the nullity of $\alpha_{11}$ constitutes the first classification criterion.

\subsection{Case A. $\alpha_{11} \neq 0$.}

Proposition 2. The nilpotent QFLA of dimension 9 with $\alpha_{11} \neq 0 \wedge \alpha_{1} \neq 0$ are nonisomorphic to the algebras with $\alpha_{11} \neq 0 \wedge$ $\alpha_{1}=0$.

Proof. If $\alpha_{11} \neq 0$, from restrictions (4a)-(4q) it can be deduced that $\alpha_{6}=\alpha_{12}=\alpha_{13}=\alpha_{14}=\alpha_{15}=\alpha_{16}=0$ and $\alpha_{7}=3 \alpha_{1}$. By computing the Jacobi equations, the family of laws is reduced to

$$
\begin{gathered}
{\left[x_{0}, x_{i}\right]=x_{i+1}, \quad 1 \leqslant i \leqslant 6,} \\
{\left[x_{1}, x_{2}\right]=\alpha_{1} x_{4}+\alpha_{2} x_{5}+\alpha_{3} x_{6}+\alpha_{4} x_{7}+\alpha_{5} x_{8}}
\end{gathered}
$$

$$
\begin{gathered}
{\left[x_{1}, x_{3}\right]=\alpha_{1} x_{5}+\alpha_{2} x_{6}+\alpha_{3} x_{7},} \\
{\left[x_{1}, x_{4}\right]=3 \alpha_{1} x_{6}+\alpha_{8} x_{7}+\alpha_{9} x_{8},} \\
{\left[x_{1}, x_{5}\right]=5 \alpha_{1} x_{7},} \\
{\left[x_{1}, x_{6}\right]=\alpha_{10} x_{7}+\alpha_{11} x_{8},} \\
{\left[x_{2}, x_{3}\right]=-2 \alpha_{1} x_{6}+\left(\alpha_{2}-\alpha_{8}\right) x_{7}-\alpha_{9} x_{8},} \\
{\left[x_{2}, x_{4}\right]=-2 \alpha_{1} x_{7},} \\
{\left[x_{2}, x_{5}\right]=-\alpha_{10} x_{7}-\alpha_{11} x_{8},} \\
{\left[x_{3}, x_{4}\right]=\alpha_{10} x_{7}+\alpha_{11} x_{8}}
\end{gathered}
$$

without restrictions derived from the Jacobi identity (4a)(4q). It can be observed that $x_{7}$ and $x_{8}$ are now central; thus the application of the elementary change of base

$$
\begin{gathered}
y_{i}=x_{i}, \quad i \neq 8, \\
y_{8}=\alpha_{10} \cdot x_{7}+\alpha_{11} \cdot x_{8}
\end{gathered}
$$

permits us to suppose that $\alpha_{10}=0$ and $\alpha_{11}=1$. Then (5f), (5i), and (5j) are simplified and the derived series is $\mathscr{D}^{1} \mathfrak{g}=\left\langle x_{2}, x_{3}, x_{4}, x_{5}, x_{6}, x_{7}, x_{8}\right\rangle, \mathscr{D}^{2} \mathfrak{g}=\left\langle-2 \alpha_{1} x_{6},\left(\alpha_{2}-\right.\right.$ $\left.\left.\alpha_{8}\right) x_{7},-\alpha_{9} x_{8},-2 \alpha_{1} x_{7}, x_{8}\right\rangle$, and so forth. Thus $\operatorname{Dim}\left[\mathscr{D}^{2} \mathfrak{g}\right]=3$ if $\alpha_{1} \neq 0$ and $\operatorname{Dim}\left[\mathscr{D}^{2} \mathfrak{g}\right] \leqslant 2$ if $\alpha_{1}=0$. Therefore the nullity of $\alpha_{1}$ constitutes a new classification criterion.

In this subsection (case $\mathrm{A}$ ), the notation to describe the parameters of the subfamily $i$ is reduced to $\mu_{i}\left(\alpha_{1}, \alpha_{2}\right.$, $\left.\alpha_{3}, \alpha_{4}, \alpha_{5}, \alpha_{7}, \alpha_{8}, \alpha_{9}\right)$ for simplification. Figure 1 shows the classification in 19 subfamilies in the case A. They are classified with the criteria summarized in Figure 2 and detailed in the following cases.

\subsubsection{Case A.1. One has $\alpha_{11} \neq 0$ and $\alpha_{1} \neq 0$.}

Proposition 3. Case A.1 permits us to suppose that $\alpha_{1}=1$.

Proof. With the elementary change of base $\mathrm{CB}$,

$$
\begin{gathered}
y_{0}=x_{0}, \\
y_{i}=\frac{x_{i}}{\alpha_{1}}, \quad 1 \leqslant i \leqslant 7, \\
y_{8}=\frac{x_{8}}{\alpha_{1}^{2}},
\end{gathered}
$$

$|\mathrm{CB}|=1 / \alpha_{1}^{9} \neq 0$ and then $\alpha_{1}=1$.

The subfamilies of laws with the structure $(5 a)-(5 j)$ are $\mu_{i}\left(1, \alpha_{2}, \alpha_{3}, \alpha_{4}, \alpha_{5}, 3, \alpha_{8}, \alpha_{9}\right)$ with $i=1,2,3$.

Let us denote, from here forward, by $\delta$ the new parameters obtained from the changes of base and $\bar{\mu}$ the Lie algebra families depending on these new parameters $\delta$ (which in general depend on the 16 parameters $\alpha$ ), in order to differentiate the new representation $\bar{\mu}_{i}\left(\delta_{j}\right)$ from the representation of the families depending in general on the 16 parameters $\mu_{i}\left(\alpha_{1}, \ldots, \alpha_{16}\right)$. 


\begin{tabular}{|c|c|c|c|c|c|c|c|c|}
\hline$\mu$ & $\alpha_{1}$ & $\alpha_{2}$ & $\alpha_{3}$ & $\alpha_{4}$ & $\alpha_{5}$ & $\alpha_{7}$ & $\alpha_{8}$ & $\alpha_{9}$ \\
\hline 1 & 1 & 0 & 0 & 0 & 0 & 3 & 1 & $\lambda$ \\
\hline 2 & 1 & 0 & 0 & 0 & 0 & 3 & 0 & 1 \\
\hline 3 & 1 & 0 & 0 & 0 & 0 & 3 & 0 & 0 \\
\hline 4 & 0 & $\lambda$ & 1 & 0 & 0 & 0 & 1 & a \\
\hline 5 & 0 & $1 / 4$ & 1 & 1 & 0 & 0 & 1 & 0 \\
\hline 6 & 0 & 1 & 1 & 1 & 0 & 0 & 0 & 0 \\
\hline 7 & 0 & $\lambda$ & 0 & 1 & 0 & 0 & 1 & 0 \\
\hline 8 & 0 & 1 & 0 & 1 & 0 & 0 & 4 & 0 \\
\hline 9 & 0 & 1 & 0 & 0 & 0 & 0 & 4 & 0 \\
\hline 10 & 0 & 1 & 0 & 1 & 0 & 0 & 0 & 0 \\
\hline 11 & 0 & 1 & 0 & 0 & 0 & 0 & 0 & 0 \\
\hline 12 & 0 & 0 & 1 & 0 & 0 & 0 & 1 & () \\
\hline 13 & 0 & 0 & 0 & 1 & 0 & 0 & 1 & 0 \\
\hline 14 & 0 & 1 & 1 & 0 & 0 & 0 & 1 & 0 \\
\hline 15 & 0 & 1 & 0 & 0 & 0 & 0 & 1 & 0 \\
\hline 16 & 0 & 0 & 1 & 1 & 0 & 0 & 0 & 0 \\
\hline 17 & 0 & 0 & 1 & 0 & 0 & 0 & 0 & 0 \\
\hline 18 & 0 & 0 & 0 & 1 & 0 & 0 & 0 & 0 \\
\hline 19 & 0 & 0 & 0 & 0 & 0 & 0 & 0 & 0 \\
\hline
\end{tabular}

Aa Classification criterion

Classifcation criterion from previous cases

Additional result

Aa Additional result from previous cases

Aa Direct result

FIGURE 1: Case A: $\alpha_{11} \neq 0$; classification of the nonisomorphic QFLA of dimension 9 (the last 7 values, $0,1,0,0,0,0,0$, common to the 19 families have been omitted from the figure for simplicity).

Proposition 4. The nilpotent QFLA of dimension 9 with $\alpha_{11} \neq 0$ and $\alpha_{1} \neq 0$ can be classified in three nonisomorphic subfamilies $\mu_{i}$ with i from 1 to 3, described in Figure 1, according to the conditions described in Figure 2.

Proof. Let us apply the change of base:

$$
\begin{gathered}
y_{0}=P_{0} x_{0}+P_{1} x_{1}+P_{2} x_{2}+P_{3} x_{3}+P_{4} x_{4} \\
+P_{5} x_{5}+P_{6} x_{6}+P_{7} x_{7}+P_{8} x_{8}, \\
y_{1}=Q_{0} x_{0}+Q_{1} x_{1}+Q_{2} x_{2}+Q_{3} x_{3}+Q_{4} x_{4} \\
+Q_{5} x_{5}+Q_{6} x_{6}+Q_{7} x_{7}+Q_{8} x_{8}, \\
y_{i+1}=\left[y_{0}, y_{i}\right], \quad 1 \leqslant i \leqslant 6, \\
y_{8}=\left[y_{1}, y_{6}\right] .
\end{gathered}
$$

The subfamilies of laws are $\bar{\mu}_{i}\left(\delta_{2}, \delta_{3}, \delta_{4}, \delta_{5}, \delta_{8}, \delta_{9}\right)$. The determinant of the change matrix is $P_{0}^{18}\left(P_{0} Q_{1}-P_{1} Q_{0}\right)^{9}$; thus $P_{0} \neq 0$ and $\left(P_{0} Q_{1}-P_{1} Q_{0}\right) \neq 0$. Since the coefficient of $y_{3}$ and $y_{4}$ in $\left[y_{1}, y_{2}\right]$ must be null, then $Q_{0}=0$ and $Q_{1}=P_{0}^{2}$. Let us apply (8) again and the only restriction is $P_{0} \neq 0$. Thus, with the coefficient identifications, the new parameters are

$$
\begin{aligned}
& \boldsymbol{\delta}_{2}=\frac{\left(-2 P_{1}+\alpha_{2} P_{0}\right)}{P_{0}^{2}}, \\
& \boldsymbol{\delta}_{3}=\frac{\left(-2 Q_{2}^{2}+4 P_{0}^{2} Q_{3}-7 P_{1} \alpha_{2} P_{0}^{3}+7 P_{1}^{2} P_{0}^{2}+\alpha_{3} P_{0}^{4}\right)}{P_{0}^{6}}, \\
& \boldsymbol{\delta}_{4}=\left(\alpha_{4} P_{0}^{6}-2 \alpha_{2}^{2} P_{0}^{5} P_{1}-2 \alpha_{2} P_{0}^{4} Q_{3}+\alpha_{2} P_{0}^{2} Q_{2}^{2}\right. \\
& -\alpha_{2} P_{0}^{5} P_{1} \alpha_{8}+2 Q_{2}^{3}-12 P_{1} \alpha_{3} P_{0}^{5} \\
& -42 P_{1} P_{0}^{3} Q_{3}-44 P_{1}^{3} P_{0}^{3}+56 P_{0}^{4} P_{1}^{2} \alpha_{2} \\
& +18 P_{1} Q_{2}^{2} P_{0}-6 P_{0}^{2} Q_{3} Q_{2}+6 P_{0}^{4} Q_{4} \\
& +6 P_{2} P_{0}^{3} Q_{2}+P_{0}^{4} P_{1}^{2} \alpha_{8}-6 P_{0}^{5} P_{3} \\
& \left.+2 \alpha_{8} P_{0}^{4} Q_{3}-Q_{2}^{2} P_{0}^{2} \alpha_{8}\right) \times\left(P_{0}^{9}\right)^{-1} \text {, } \\
& \boldsymbol{\delta}_{5}=\left(2 P_{0} P_{1} Q_{3} Q_{2}+2 P_{2} \alpha_{2} P_{0}^{3} Q_{2}+6 P_{1}^{2} \alpha_{3} P_{0}^{4}\right. \\
& -2 P_{2} P_{0} Q_{2}^{2}-2 P_{0}^{2} Q_{4} Q_{2}+6 P_{0}^{4} P_{3} P_{1} \\
& -8 P_{1}^{2} Q_{2}^{2}-2 P_{0} P_{1} Q_{2}^{2} \alpha_{2}-4 P_{1} P_{2} P_{0}^{2} Q_{2} \\
& +2 P_{2} P_{0}^{3} Q_{3}+2 P_{3} P_{0}^{3} Q_{2}-4 P_{1} P_{0}^{3} Q_{4} \\
& +20 P_{0}^{2} P_{1}^{2} Q_{3}-Q_{2}^{2} P_{0}^{2} \alpha_{9}+2 Q_{3} P_{0}^{4} \alpha_{9} \\
& +P_{1} Q_{2}^{2} P_{0} \alpha_{8}-P_{1} P_{0}^{5} \alpha_{4}+P_{0}^{2} Q_{3}^{2}+2 P_{0}^{4} Q_{5} \\
& +P_{0}^{6} \alpha_{5}+2 P_{1} P_{0}^{3} Q_{3} \alpha_{2}-2 P_{1} \alpha_{8} P_{0}^{3} Q_{3} \\
& +\alpha_{2} P_{0}^{4} P_{1}^{2} \alpha_{8}-\alpha_{2} P_{0}^{5} P_{1} \alpha_{9}-2 P_{0}^{5} P_{3} \alpha_{2} \\
& -2 P_{0}^{5} P_{4}+15 P_{0}^{2} P_{1}^{4}-P_{0}^{4} P_{2}^{2}+P_{0}^{4} P_{1}^{2} \alpha_{9} \\
& \left.-20 P_{0}^{3} P_{1}^{3} \alpha_{2}-P_{0}^{3} P_{1}^{3} \alpha_{8}\right) \times\left(P_{0}^{10}\right)^{-1}, \\
& \boldsymbol{\delta}_{8}=\frac{\left(-20 P_{1}+\alpha_{8} P_{0}\right)}{P_{0}^{2}}, \\
& \boldsymbol{\delta}_{9}=\frac{\left(8 P_{1}^{2} P_{0}^{2}-P_{0}^{3} P_{1} \alpha_{8}+P_{0}^{4} \alpha_{9}+2 P_{0}^{2} Q_{3}+2 P_{1} \alpha_{2} P_{0}^{3}-Q_{2}^{2}\right)}{P_{0}^{6}} .
\end{aligned}
$$

Let us select $P_{1}, Q_{3}, P_{3}$, and $P_{4}$ appropriately and the subfamilies of laws result in $\bar{\mu}_{i}\left(0,0,0,0, \delta_{8}, \delta_{9}\right)$

$$
\begin{gathered}
{\left[y_{0}, y_{i}\right]=y_{i+1}, \quad 1 \leqslant i \leqslant 6,} \\
{\left[y_{1}, y_{2}\right]=y_{4},} \\
{\left[y_{1}, y_{3}\right]=y_{5},} \\
{\left[y_{1}, y_{4}\right]=3 y_{6}+\delta_{8} y_{7}+\delta_{9} y_{8},}
\end{gathered}
$$




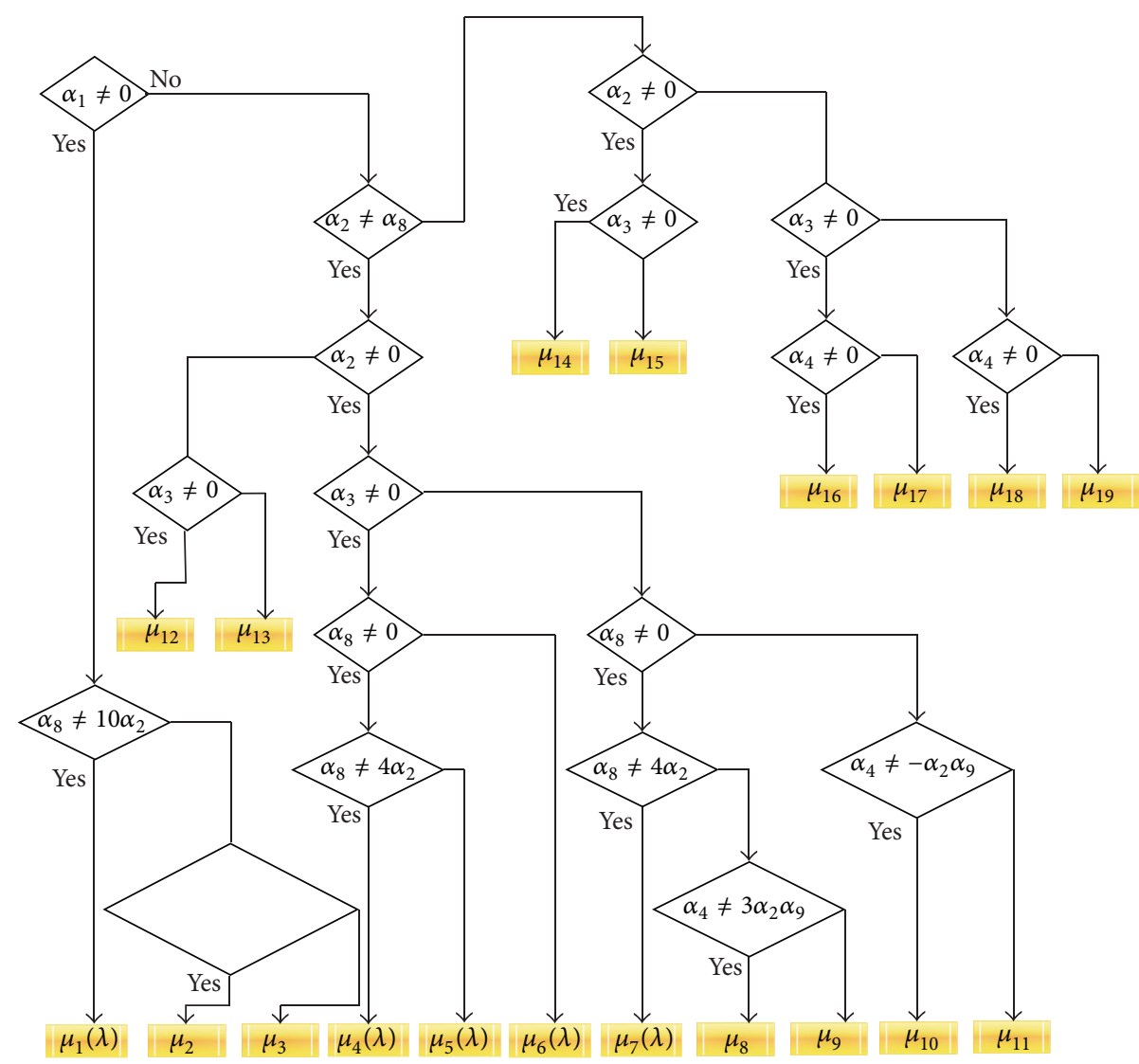

FIgURE 2: Case A: $\alpha_{11} \neq 0$; nonisomorphic subfamilies determination.

$$
\begin{gathered}
{\left[y_{1}, y_{5}\right]=5 y_{7},} \\
{\left[y_{1}, y_{6}\right]=y_{8},} \\
{\left[y_{2}, y_{3}\right]=-2 y_{6}-\delta_{8} y_{7}-\delta_{9} y_{8},} \\
{\left[y_{2}, y_{4}\right]=-2 y_{7},} \\
{\left[y_{2}, y_{5}\right]=-y_{8},} \\
{\left[y_{3}, y_{4}\right]=y_{8} .}
\end{gathered}
$$

The nullity of $-10 \alpha_{2}+\alpha_{8}$ and the nullity of $31 \alpha_{2}^{2}+8 \alpha_{9}-4 \alpha_{3}-4 \alpha_{2} \alpha_{8}$ are invariants. It can be proved by substituting (9) and (13) in the expressions $-10 \delta_{2}+\delta_{8}$ and $31 \delta_{2}^{2}+8 \delta_{9}-4 \delta_{3}-4 \delta_{2} \delta_{8}$; then $P_{0}$ is in the denominator and since it must be nonnull, the nullity of those expressions is invariant for the change (8). Then four subcases are determined by the nullity of $\delta_{8}$ and $\delta_{9}$. If $\delta_{8} \neq 0$, the subcases corresponding to $\delta_{9}$ nullity or nonnullity can be reduced to a subfamily of algebras $\boldsymbol{\mu}_{1}^{\lambda}(\mathbf{1}, \mathbf{0}, \mathbf{0}, \mathbf{0}, \mathbf{0}, \mathbf{0}, \mathbf{3}, \mathbf{1}, \boldsymbol{\lambda}, \mathbf{0}, \mathbf{1})$ with one parameter $\lambda \in \mathbf{C}$, with $\lambda=(1 / 8)\left(-31 \alpha_{2}^{2}+8 \alpha_{9}+4 \alpha_{3}+4 \alpha_{2} \alpha_{8}\right) /\left(-31 \alpha_{2}+31 \alpha_{8}\right)^{2}$. If $\delta_{8}=$ 0 , in the subcase corresponding to $\delta_{9} \neq 0, P_{0}$ can be selected as $\left(-18 \alpha_{2}^{2}+16 \alpha_{9}-8 \alpha_{3}\right)^{1 / 2} / 4$ since $\alpha_{9} \neq\left(-31 \alpha_{2}^{2}+4 \alpha_{3}+4 \alpha_{2} \alpha_{8}\right) / 8$ and $\alpha_{8}=10 \alpha_{2}$ imply $-9 \alpha_{2}^{2}+8 \alpha_{9}-4 \alpha_{3} \neq 0$.
Thus $\delta_{9}=1$, and the algebra is described by $\boldsymbol{\mu}_{2}(\mathbf{1}, \mathbf{0}, \mathbf{0}, \mathbf{0}, \mathbf{0}, \mathbf{0}, \mathbf{3}, \mathbf{0}, \mathbf{1}, \mathbf{0}, \mathbf{1})$. Finally, the forth subcase is the algebra $\boldsymbol{\mu}_{3}(\mathbf{1}, \mathbf{0}, \mathbf{0}, \mathbf{0}, \mathbf{0}, \mathbf{0}, \mathbf{3}, \mathbf{0}, \mathbf{0}, \mathbf{0}, \mathbf{1})$.

2.3.2. Case A.2. One has $\alpha_{11} \neq 0$ and $\alpha_{1}=0$.

Proposition 5. The nilpotent QFLA of dimension 9 with $\alpha_{11} \neq 0, \alpha_{1}=0$, and $\alpha_{2}=\alpha_{8}$ are nonisomorphic to the algebras with $\alpha_{11} \neq 0, \alpha_{1}=0$, and $\alpha_{2} \neq \alpha_{8}$.

Proof. The derived series is $\mathscr{D}^{1} \mathfrak{g}=\left\langle x_{2}, x_{3}, x_{4}, x_{5}, x_{6}, x_{7}, x_{8}\right\rangle$, $\mathscr{D}^{2} \mathfrak{g}=\left\langle\left(\alpha_{2}-\alpha_{8}\right) x_{7}, x_{8}\right\rangle$, and so forth; thus $\operatorname{Dim}\left[\mathscr{D}^{2} \mathfrak{g}\right]=2$ if $\alpha_{2} \neq \alpha_{8}$ and $\operatorname{Dim}\left[\mathscr{D}^{2} \mathfrak{g}\right]=1$ if $\alpha_{2}=\alpha_{8}$. The nullity of $\alpha_{2}-\alpha_{8}$ constitutes a new classification criterion.

Proposition 6. The nilpotent QFLA of dimension 9 with $\alpha_{11} \neq 0, \alpha_{1}=0$, and $\alpha_{2} \neq \alpha_{8}$ can be classified in ten nonisomorphic subfamilies $\mu_{j}$ with $j$ from 4 to 13 , described in Figure 1, according to the conditions described in Figure 2.

Proof. Let us apply the change of base (8). The subfamilies of laws are $\bar{\mu}_{j}\left(\delta_{2}, \delta_{3}, \delta_{4}, \delta_{5}, \delta_{8}, \delta_{9}\right)$ and the restrictions $P_{0} \neq 0$ and $Q_{1} \neq 0$. Thus, with the coefficient identifications, the new parameters are

$$
\delta_{2}=\frac{Q_{1} \alpha_{2}}{P_{0}^{3}}
$$




$$
\begin{gathered}
\delta_{3}=\frac{Q_{1} \alpha_{3}}{P_{0}^{3}} \\
\delta_{4}=-\left(2 Q_{1}^{2} P_{1} \alpha_{2}^{2}+2 Q_{3} P_{0} Q_{1} \alpha_{2}-Q_{2}^{2} P_{0} \alpha_{2}\right. \\
+\alpha_{2} Q_{1}^{2} P_{1} \alpha_{8}-Q_{1}^{2} P_{0} \alpha_{4} \\
\left.-2 Q_{1} P_{0} Q_{3} \alpha_{8}+P_{0} Q_{2}^{2} \alpha_{8}\right) \times\left(Q_{1} P_{0}^{6}\right)^{-1} \\
\delta_{5}=\left(-\alpha_{2} Q_{1}^{2} P_{1} P_{0} \alpha_{9}-2 \alpha_{2} Q_{1}^{2} P_{3} P_{0}+\alpha_{2} Q_{1}^{2} P_{1}^{2} \alpha_{8}\right. \\
+2 P_{0} P_{2} Q_{1} \alpha_{2} Q_{2}-P_{1} P_{0} \alpha_{4} Q_{1}^{2}-2 P_{1} P_{0} Q_{1} Q_{3} \alpha_{8} \\
-2 P_{1} P_{0} \alpha_{2} Q_{2}^{2}+P_{1} P_{0} Q_{2}^{2} \alpha_{8}+2 P_{1} P_{0} \alpha_{2} Q_{1} Q_{3} \\
+Q_{1}^{2} P_{0}^{2} \alpha_{5}+2 Q_{1} P_{0}^{2} Q_{3} \alpha_{9}+2 Q_{5} P_{0}^{2} Q_{1} \\
\left.-Q_{2}^{2} P_{0}^{2} \alpha_{9}-2 Q_{4} P_{0}^{2} Q_{2}+Q_{3}^{2} P_{0}^{2}\right) \times\left(Q_{1}^{2} P_{0}^{6}\right)^{-1} \\
\delta_{8}=\frac{\alpha_{8} Q_{1}}{P_{0}^{3}}, \\
\delta_{9} \frac{\left(-Q_{1}^{2} P_{1} \alpha_{8}+Q_{1}^{2} P_{0} \alpha_{9}+2 P_{0} Q_{3} Q_{1}+2 Q_{1}^{2} P_{1} \alpha_{2}-P_{0} Q_{2}^{2}\right)}{P_{1}^{3} Q_{1}^{2}}
\end{gathered}
$$

Selecting $Q_{5}$ and $Q_{3}$ appropriately the subfamilies of laws result in $\bar{\mu}_{j}\left(\delta_{2}, \delta_{3}, \delta_{4}, 0, \delta_{8}, 0\right)$. From (16), (17), and (20) the invariance of the nullities of $\alpha_{2}, \alpha_{3}$, and $\alpha_{8}$ is clear. The nullity of $4 \alpha_{2}-\alpha_{8}$ is also invariant. It can be proved by substituting (16) and (20) in the expression $4 \delta_{2}-\delta_{8}$ considering the change restrictions. If $\boldsymbol{\alpha}_{11} \neq \mathbf{0}, \alpha_{1}=\mathbf{0}, \boldsymbol{\alpha}_{2} \neq \boldsymbol{\alpha}_{8}, \boldsymbol{\alpha}_{2} \neq \mathbf{0}, \alpha_{3} \neq \mathbf{0}, \alpha_{8} \neq \mathbf{0}$, and $\alpha_{8} \neq 4 \alpha_{2}$, selecting $Q_{1}=P_{0}^{3} / \alpha_{8} \neq 0, P_{0}=\alpha_{3} / \alpha_{8} \neq 0$, and $P_{1}=\alpha_{3}\left(-\alpha_{8} \alpha_{9}+\alpha_{4}+\alpha_{2} \alpha_{9}\right) /\left(\alpha_{8}^{2}\left(-\alpha_{8}+4 \alpha_{2}\right)\right)$, the subfamily is $\boldsymbol{\mu}_{4}^{\lambda}(\mathbf{0}, \boldsymbol{\lambda}, \mathbf{1}, \mathbf{0}, \mathbf{0}, \mathbf{0}, \mathbf{1}, \mathbf{0})$, with $\delta_{2}=\boldsymbol{\alpha}_{2} / \boldsymbol{\alpha}_{8}=$ $\lambda \in \mathbf{C}-\{\mathbf{0}, \mathbf{1}, \mathbf{1} / \mathbf{4}\}$. If $\boldsymbol{\alpha}_{11} \neq \mathbf{0}, \alpha_{1}=\mathbf{0}, \boldsymbol{\alpha}_{2} \neq \boldsymbol{\alpha}_{8}, \boldsymbol{\alpha}_{2} \neq \mathbf{0}$, $\alpha_{3} \neq \mathbf{0}, \alpha_{8} \neq \mathbf{0}$, and $\alpha_{8}=\mathbf{4} \boldsymbol{\alpha}_{2}$, then $\delta_{4}$ is invariant, and the subfamily is $\boldsymbol{\mu}_{5}^{\lambda}(\mathbf{0}, \mathbf{1} / \mathbf{4}, \mathbf{1}, \boldsymbol{\lambda}, \mathbf{0}, \mathbf{0}, \mathbf{1}, \mathbf{0}), \lambda \in \mathrm{C}$. If $\boldsymbol{\alpha}_{11} \neq \mathbf{0}$, $\alpha_{1}=\mathbf{0}, \alpha_{2} \neq \boldsymbol{\alpha}_{8}, \boldsymbol{\alpha}_{2} \neq \mathbf{0}, \alpha_{3} \neq \mathbf{0}$, and $\boldsymbol{\alpha}_{8}=\mathbf{0}$, selecting $Q_{1}=P_{0}^{4} / \alpha_{3} \neq 0$ and $P_{0}=\alpha_{3} / \alpha_{2} \neq 0$, the subfamily is $\boldsymbol{\mu}_{6}^{\lambda}(\mathbf{0}, \mathbf{1}, \mathbf{1}, \boldsymbol{\lambda}, \mathbf{0}, \mathbf{0}, \mathbf{0}, \mathbf{0}), \boldsymbol{\lambda} \in \mathrm{C}$. If $\boldsymbol{\alpha}_{11} \neq \mathbf{0}, \alpha_{1}=\mathbf{0}, \alpha_{2} \neq \boldsymbol{\alpha}_{8}$, $\alpha_{2} \neq \mathbf{0}, \alpha_{3}=\mathbf{0}, \boldsymbol{\alpha}_{8} \neq \mathbf{0}$, and $\boldsymbol{\alpha}_{8} \neq \mathbf{4} \boldsymbol{\alpha}_{2}$, selecting $Q_{1}=P_{0}^{3} / \alpha_{8} \neq 0$ and $P_{1}=P_{0}\left(-\alpha_{9} \alpha_{8}+\alpha_{2} \alpha_{9}+\alpha_{4}-P_{0}^{2} \alpha_{8}\right) /\left(\alpha_{8}\left(-\alpha_{8}+4 \alpha_{2}\right)\right)$, the subfamily is $\boldsymbol{\mu}_{7}^{\lambda}(\mathbf{0}, \boldsymbol{\lambda}, \mathbf{0}, \mathbf{1}, \mathbf{0}, \mathbf{0}, \mathbf{1}, \mathbf{0}), \lambda \in \mathbf{C}-\{\mathbf{0}, \mathbf{1}, \mathbf{1} / \mathbf{4}\}$. If $\boldsymbol{\alpha}_{11} \neq \mathbf{0}, \alpha_{1}=\mathbf{0}, \boldsymbol{\alpha}_{2} \neq \boldsymbol{\alpha}_{8}, \alpha_{2} \neq \mathbf{0}, \alpha_{3}=\mathbf{0}, \boldsymbol{\alpha}_{8} \neq \mathbf{0}$, and $\boldsymbol{\alpha}_{8}=$ $4 \alpha_{2}$, selecting $Q_{1}=P_{0}^{3} / \alpha_{2}$, the nullity of $-\alpha_{4}+3 \alpha_{2} \alpha_{9}$ is invariant. If $\alpha_{4} \neq 3 \alpha_{2} \alpha_{9}$, selecting $P_{0}^{2}=-\alpha_{2}\left(-\alpha_{4}+3 \alpha_{2} \alpha_{9}\right) / \alpha_{2}^{2}$, the subfamily is $\boldsymbol{\mu}_{8}(\mathbf{0}, \mathbf{1}, \mathbf{0}, \mathbf{1}, \mathbf{0}, \mathbf{0}, \mathbf{4}, \mathbf{0})$, else the subfamily is $\boldsymbol{\mu}_{9}(\mathbf{0}, \mathbf{1}, \mathbf{0}, \mathbf{0}, \mathbf{0}, \mathbf{0}, \mathbf{4}, \mathbf{0})$. If $\boldsymbol{\alpha}_{11} \neq \mathbf{0}, \alpha_{1}=\mathbf{0}, \alpha_{2} \neq \boldsymbol{\alpha}_{8}, \boldsymbol{\alpha}_{2} \neq \mathbf{0}, \alpha_{3}=$ $\mathbf{0}$, and $\boldsymbol{\alpha}_{8}=\mathbf{0}$, selecting $Q_{1}=P_{0}^{3} / \alpha_{2}$, the nullity of $\alpha_{2} \alpha_{9}+\alpha_{4}$ is invariant. If $\boldsymbol{\alpha}_{4} \neq-\boldsymbol{\alpha}_{2} \boldsymbol{\alpha}_{9}$, selecting $P_{0}^{2}=\alpha_{2}\left(\alpha_{2} \alpha_{9}+\alpha_{4}\right) / \alpha_{2}^{2} \neq 0$, the subfamily is $\boldsymbol{\mu}_{10}(\mathbf{0}, \mathbf{1}, \mathbf{0}, \mathbf{1}, \mathbf{0}, \mathbf{0}, \mathbf{0}, \mathbf{0})$; else with $Q_{1}=P_{0}^{3} / \alpha_{2}$ the subfamily is $\boldsymbol{\mu}_{11}(\mathbf{0}, \mathbf{1}, \mathbf{0}, \mathbf{0}, \mathbf{0}, \mathbf{0}, \mathbf{0}, \mathbf{0})$. If $\boldsymbol{\alpha}_{11} \neq \mathbf{0}, \alpha_{1}=\mathbf{0}$, $\alpha_{2} \neq \boldsymbol{\alpha}_{8}, \boldsymbol{\alpha}_{2}=\mathbf{0}$, and $\alpha_{3} \neq \mathbf{0}$, selecting $Q_{1}=P_{0}^{4} / \alpha_{3} \neq 0, P_{0}=$ $\alpha_{3} / \alpha_{8} \neq 0$, and $P_{1}=-\alpha_{3}\left(\alpha_{4}-\alpha_{9} \alpha_{8}\right) / \alpha_{8}^{3}$, the subfamily is $\boldsymbol{\mu}_{12}(\mathbf{0}, \mathbf{0}, \mathbf{1}, \mathbf{0}, \mathbf{0}, \mathbf{0}, \mathbf{1}, \mathbf{0})$. If $\boldsymbol{\alpha}_{11} \neq \mathbf{0}, \alpha_{1}=\mathbf{0}, \alpha_{2} \neq \boldsymbol{\alpha}_{8}, \boldsymbol{\alpha}_{2}=\mathbf{0}$, and $\alpha_{3}=\mathbf{0}$, selecting $Q_{1}=P_{0}^{3} / \alpha_{8} \neq 0$ and $P_{1}=P_{0}\left(-\alpha_{4}+\alpha_{9} \alpha_{8}+\right.$ $\left.P_{0}^{2} \alpha_{8}\right) / \alpha_{8}^{2} \neq 0$, the subfamily is $\boldsymbol{\mu}_{13}(\mathbf{0}, \mathbf{0}, \mathbf{0}, \mathbf{1}, \mathbf{0}, \mathbf{0}, \mathbf{1}, \mathbf{0})$.

Proposition 7. The nilpotent QFLA of dimension 9 with $\alpha_{11} \neq 0, \alpha_{1}=0$, and $\alpha_{2}=\alpha_{8}$ can be classified in six nonisomorphic subfamilies $\mu_{k}$ with $k$ from 14 to 19 , described in Figure 1, according to the conditions described in Figure 2.

Proof. Let us apply the change of base (8). The subfamilies of laws are $\bar{\mu}_{k}\left(\delta_{2}, \delta_{3}, \delta_{4}, \delta_{5}, \delta_{8}, \delta_{9}\right)$ and the restrictions $P_{0} \neq 0$ and $Q_{1} \neq 0$. Thus, with the coefficient identifications, the new parameters are

$$
\begin{gathered}
\delta_{2}=\frac{Q_{1} \alpha_{2}}{P_{0}^{3}}, \\
\delta_{3}=\frac{Q_{1} \alpha_{3}}{P_{0}^{4}}, \\
\delta_{4}=\frac{Q_{1}\left(-3 \alpha_{2}^{2} P_{1}+P_{0} \alpha_{4}\right)}{P_{0}^{6}}, \\
\delta_{5}=\left(-\alpha_{2} Q_{1}^{2} P_{1} P_{0} \alpha_{9}-2 \alpha_{2} Q_{1}^{2} P_{3} P_{0}+\alpha_{2}^{2} Q_{1}^{2} P_{1}^{2}\right. \\
+2 P_{2} P_{0} Q_{1} \alpha_{2} Q_{2}-P_{0} Q_{1}^{2} P_{1} \alpha_{4}+Q_{1}^{2} P_{0}^{2} \alpha_{5} \\
+2 Q_{1} P_{0}^{2} Q_{3} \alpha_{9}+2 Q_{5} P_{0}^{2} Q_{1}-Q_{2}^{2} P_{0}^{2} \alpha_{9} \\
\left.-2 Q_{4} P_{0}^{2} Q_{2}-P_{0} P_{1} Q_{2}^{2} \alpha_{2}+Q_{3}^{2} P_{0}^{2}\right) \times\left(Q_{1}^{2} P_{0}^{6}\right)^{-1}, \\
\delta_{9}=\frac{\left(P_{1} Q_{1}^{2} \alpha_{2}+Q_{1}^{2} P_{0} \alpha_{9}+2 P_{0} Q_{3} Q_{1}-P_{0} Q_{2}^{2}\right)}{\left(P_{0}^{3} Q_{1}^{2}\right)} .
\end{gathered}
$$

Let us select $Q_{5}$ and $Q_{3}$ appropriately and the subfamilies of laws result in $\bar{\mu}_{k}\left(\delta_{2}, \delta_{3}, \delta_{4}, 0,0\right)$. From (22), (23), and (24) the invariance of the nullities of $\alpha_{2}, \alpha_{3}$, and $\alpha_{4}$ is clear. If $\boldsymbol{\alpha}_{11} \neq \mathbf{0}$, $\alpha_{1}=\mathbf{0}, \alpha_{2}=\boldsymbol{\alpha}_{8}, \boldsymbol{\alpha}_{2} \neq \mathbf{0}$, and $\alpha_{3} \neq \mathbf{0}$, selecting $Q_{1}=P_{0}^{3} / \alpha_{2} \neq 0$, $P_{0}=\alpha_{3} / \alpha_{2} \neq 0$, and $P_{1}=\alpha_{3} \alpha_{4} /\left(3 \alpha_{2}^{3}\right)$, the subfamily is $\boldsymbol{\mu}_{14}(\mathbf{0}, \mathbf{1}, \mathbf{1}, \mathbf{0}, \mathbf{0}, \mathbf{0}, \mathbf{1}, \mathbf{0})$. If $\boldsymbol{\alpha}_{11} \neq \mathbf{0}, \alpha_{1}=\mathbf{0}, \alpha_{2}=\boldsymbol{\alpha}_{8}, \boldsymbol{\alpha}_{2} \neq \mathbf{0}$, and $\alpha_{3}=\mathbf{0}$, selecting $Q_{1}=P_{0}^{3} / \alpha_{2} \neq 0$ and $P_{1}=P_{0} \alpha_{4} /\left(3 \alpha_{2}^{2}\right)$, the subfamily is $\boldsymbol{\mu}_{15}(\mathbf{0}, \mathbf{1}, \mathbf{0}, \mathbf{0}, \mathbf{0}, \mathbf{0}, \mathbf{1}, \mathbf{0})$. If $\boldsymbol{\alpha}_{11} \neq \mathbf{0} \wedge \boldsymbol{\alpha}_{1}=\mathbf{0}$, $\alpha_{2}=\boldsymbol{\alpha}_{8}, \boldsymbol{\alpha}_{2}=\mathbf{0}$, and $\alpha_{3} \neq \mathbf{0}$, selecting $Q_{1}=P_{0}^{4} / \alpha_{3} \neq 0$, the nullity of $\alpha_{4}$ is invariant. If $\alpha_{4} \neq 0$ and $P_{0}=\alpha_{4} / \alpha_{3} \neq 0$, the subfamily is $\boldsymbol{\mu}_{16}(\mathbf{0}, \mathbf{0}, \mathbf{1}, \mathbf{1}, \mathbf{0}, \mathbf{0}, \mathbf{0}, \mathbf{0})$. If $\alpha_{4}=0$ the subfamily is $\boldsymbol{\mu}_{17}(\mathbf{0}, \mathbf{0}, \mathbf{1}, \mathbf{0}, \mathbf{0}, \mathbf{0}, \mathbf{0}, \mathbf{0})$. If $\boldsymbol{\alpha}_{11} \neq \mathbf{0}, \alpha_{1}=\mathbf{0}, \alpha_{2}=\boldsymbol{\alpha}_{8}, \boldsymbol{\alpha}_{2}=\mathbf{0}$, and $\alpha_{3}=\mathbf{0}$, selecting $Q_{1}=P_{0}^{5} / \alpha_{4} \neq 0$, the subfamilies are $\boldsymbol{\mu}_{18}(\mathbf{0}, \mathbf{0}, \mathbf{0}, \mathbf{1}, \mathbf{0}, \mathbf{0}, \mathbf{0}, \mathbf{0})$ with $\alpha_{4} \neq 0$, and $\boldsymbol{\mu}_{19}(\mathbf{0}, \mathbf{0}, \mathbf{0}, \mathbf{0}, \mathbf{0}, \mathbf{0}, \mathbf{0}, \mathbf{0})$ with $\alpha_{4}=0$.

\subsection{Case B. One has $\alpha_{11}=0$.}

Proposition 8. The nilpotent QFLA of dimension 9 and $\alpha_{11}=$ 0 and $\alpha_{9} \neq 0$ are nonisomorphic to the algebras with $\alpha_{11}=0$ and $\alpha_{9}=0$.

Proof. For the family described by (3a)-(3o) and (4a)-(4q), $\mathscr{C}^{3} \mathfrak{g}=\left\langle x_{4}, x_{5}, x_{6}, x_{7}, \alpha_{9} x_{8}\right\rangle$; therefore its dimension is $\operatorname{Dim}\left[\mathscr{C}^{3} \mathfrak{g}\right]=5$, if $\alpha_{9} \neq 0$ or $\operatorname{Dim}\left[\mathscr{C}^{3} \mathfrak{g}\right]=4$, if $\alpha_{9}=0$, and the nullity of $\alpha_{9}$ constitutes a new classification criterion. 


\subsubsection{Case B.1. One has $\alpha_{11}=0$ and $\alpha_{9} \neq 0$.}

Proposition 9. The nilpotent QFLA of dimension 9 with $\alpha_{11}=$ $0, \alpha_{9} \neq 0$, and $\alpha_{6} \neq 0$ are nonisomorphic to the algebras with $\alpha_{11}=0, \alpha_{9} \neq 0$, and $\alpha_{6}=0$.

Proof. If $\alpha_{9} \neq 0$, it can be deduced that $\alpha_{12}=\alpha_{13}=\alpha_{14}=0$. By computing the Jacobi equations, the family of laws is reduced to

$$
\begin{gathered}
{\left[x_{0}, x_{i}\right]=x_{i+1}, \quad 1 \leqslant i \leqslant 6} \\
{\left[x_{1}, x_{2}\right]=\alpha_{1} x_{4}+\alpha_{2} x_{5}+\alpha_{3} x_{6}+\alpha_{4} x_{7}+\alpha_{5} x_{8}} \\
{\left[x_{1}, x_{3}\right]=\alpha_{1} x_{5}+\alpha_{2} x_{6}+\alpha_{3} x_{7}} \\
{\left[x_{1}, x_{4}\right]=\alpha_{6} x_{5}+\alpha_{7} x_{6}+\alpha_{8} x_{7}+\alpha_{9} x_{8}} \\
{\left[x_{1}, x_{5}\right]=2 \alpha_{6} x_{6}+\left(2 \alpha_{7}-\alpha_{1}\right) x_{7}} \\
{\left[x_{1}, x_{6}\right]=\alpha_{10} x_{7}} \\
{\left[x_{2}, x_{8}\right]=\alpha_{15} x_{6}+\alpha_{16} x_{7}} \\
-\alpha_{6} x_{5}+\left(\alpha_{1}-\alpha_{7}\right) x_{6}+\left(\alpha_{2}-\alpha_{8}\right) x_{7}-\alpha_{9} x_{8} \\
{\left[x_{2}, x_{4}\right]=-\alpha_{6} x_{6}+\left(\alpha_{1}-\alpha_{7}\right) x_{7}} \\
{\left[x_{2}, x_{5}\right]=\left(2 \alpha_{6}-\alpha_{10}\right) x_{7}} \\
{\left[x_{2}, x_{8}\right]=\alpha_{15} x_{7}} \\
{\left[x_{3}, x_{4}\right]=\left(-3 \alpha_{6}+\alpha_{10}\right) x_{7}}
\end{gathered}
$$

with two restrictions

$$
\begin{gathered}
-2 \alpha_{6}^{2}-\alpha_{9} \alpha_{15}=0 \\
-4 \alpha_{1} \alpha_{6}+3 \alpha_{1} \alpha_{10}-2 \alpha_{6} \alpha_{7}-\alpha_{7} \alpha_{10}-\alpha_{9} \alpha_{16}=0 .
\end{gathered}
$$

Since $\alpha_{9} \neq 0$, the application of the elementary change of base $\mathrm{CB}$

$$
\begin{aligned}
y_{0}= & y_{1} \\
y_{i}= & \frac{x_{i}}{\alpha_{9}}, \quad 1 \leqslant i \leqslant n-1 \\
& \text { with }|\mathrm{CB}|=\frac{1}{\alpha_{9}^{8}} \neq 0
\end{aligned}
$$

permits us to suppose that $\alpha_{9}=1$. Then from (39), $\alpha_{15}=$ $-2 \alpha_{6}^{2}$ and $\alpha_{16}=-4 \alpha_{1} \alpha_{6}+3 \alpha_{1} \alpha_{10}-2 \alpha_{6} \alpha_{7}-\alpha_{7} \alpha_{10}$. This implies that (33) and (37) are changed to $\left[x_{1}, x_{8}\right]=-2 \alpha_{6}^{2} x_{6}+$ $\left(\alpha_{1}\left(-4 \alpha_{6}+3 \alpha_{10}\right)-\alpha_{7}\left(2 \alpha_{6}+\alpha_{10}\right)\right) x_{7}$ and $\left[x_{2}, x_{8}\right]=$ $-2 \alpha_{6}^{2} x_{7}$, respectively, and the subfamily of laws $\mu_{l}\left(\alpha_{1}, \alpha_{2}, \alpha_{3}\right.$, $\left.\alpha_{4}, \alpha_{5}, \alpha_{6}, \alpha_{7}, \alpha_{8}, 1, \alpha_{10}, 0,0,0,0,0,0\right)$, with $l$ from 20 to 63, has no restrictions $(4 \mathrm{a})-(4 \mathrm{q})$. Its derived series is $\mathscr{D}^{1} \mathfrak{g}=$ $\left\langle x_{2}, x_{3}, x_{4}, x_{5}, x_{6}, x_{7}, x_{8}\right\rangle, \mathscr{D}^{2} \mathfrak{g}=\left\langle-\alpha_{6} x_{5}+\left(\alpha_{1}-\alpha_{7}\right) x_{6}+\left(\alpha_{2}-\right.\right.$ $\left.\alpha_{8}\right) x_{7}, x_{8},-\alpha_{6} x_{6}+\left(\alpha_{1}-\alpha_{7}\right) x_{7},\left(2 \alpha_{6}-\alpha_{10}\right) x_{7},-2 \alpha_{6}^{2} x_{7},\left(-3 \alpha_{6}+\right.$ $\left.\left.\alpha_{10}\right) x_{7}\right\rangle$, and so forth. Thus $\operatorname{Dim}\left[\mathscr{D}^{2} \mathfrak{g}\right]=4$ if $\alpha_{6} \neq 0$ and $\operatorname{Dim}\left[\mathscr{D}^{2} \mathfrak{g}\right] \leqslant 3$ if $\alpha_{6}=0$. Therefore the nullity of $\alpha_{6}$ constitutes a new classification criterion.
Case B.1.1: $\alpha_{11}=0, \alpha_{9} \neq 0$ and $\alpha_{6} \neq 0$. Figure 3 provides the classification in 18 subfamilies in this case.

Case B.1.2: $\alpha_{11}=0, \alpha_{9} \neq 0$, and $\alpha_{6}=0$. Figure 4 provides the classification in 26 subfamilies in this case.

2.4.2. Case B.2. One has $\alpha_{11}=0$ and $\alpha_{9}=0$.

The restrictions in the family $(4 a)-(4 q)$ are reduced to

$$
\begin{gathered}
\alpha_{5} \alpha_{12}=0 \\
\alpha_{6} \alpha_{12}=0 \\
\alpha_{6} \alpha_{13}=0 \\
\alpha_{10} \alpha_{12}=0 \\
\alpha_{12}\left(\alpha_{1}-\alpha_{7}\right)=0 \\
\alpha_{5} \alpha_{13}-2 \alpha_{6}^{2}=0 \\
2\left(\alpha_{2}-\alpha_{8}\right) \alpha_{12}+3\left(\alpha_{1}-\alpha_{7}\right) \alpha_{13}+2\left(\alpha_{6}-\alpha_{10}\right) \alpha_{14}=0 \\
\alpha_{5} \alpha_{14}-2\left(2 \alpha_{1}+\alpha_{7}\right) \alpha_{6}-\alpha_{9} \alpha_{16}+\left(3 \alpha_{1}-\alpha_{7}\right) \alpha_{10}=0
\end{gathered}
$$

Proposition 10. The nilpotent QFLA of dimension 9 with $\alpha_{11}=0, \alpha_{9}=0$, and $\alpha_{5} \neq 0$ are nonisomorphic to the algebras with $\alpha_{11}=0, \alpha_{9}=0$, and $\alpha_{5}=0$.

Proof. Equations (43) and (46) imply that $\alpha_{6}=0$. By computing the Jacobi equations, the subfamily of laws is $\mu_{m}\left(\alpha_{1}, \alpha_{2}, \alpha_{3}, \alpha_{4}, \alpha_{5}, 0, \alpha_{7}, \alpha_{8}, 0, \alpha_{10}, 0, \alpha_{12}, \alpha_{13}, \alpha_{14}, \alpha_{15}, \alpha_{16}\right)$, with $m$ from 64 to 263 , and the restrictions are reduced to 6 . Its descending central series is $\mathscr{C}^{1} \mathfrak{g}=$ $\left\langle x_{2}, x_{3}, x_{4}, x_{5}, x_{6}, x_{7}, \alpha_{5} x_{8}\right\rangle$, and so forth. Thus the nullity of $\alpha_{5}$ constitutes a new classification criterion.

An exhaustive and extensive process of analysis with the same methodology shown in the previous subsections leads to the final subclassification, which is summarized in the following Figures.

Case B.2.1: $\alpha_{11}=0, \alpha_{9}=0$, and $\alpha_{5} \neq 0$. Figure 5 provides the classification in 55 subfamilies in this case.

Case B.2.2: $\alpha_{11}=0, \alpha_{9}=0$, and $\alpha_{5}=0$. Figures 6 and 7 provide the classification in 145 subfamilies in this case.

\section{Concluding Remarks}

Computational aid has been indispensable in this piece of research. A PC Pentium 4 of $2.4 \mathrm{GHz}$ and the programming language Maple 6 have been used in the process. The library modules developed represent approximately 12,000 lines of code. In some cases, in this massive application of computational resources and looking for the simplification of some laws, procedures that perhaps can be considered of "inverse engineering" have been used in order to find some very complex changes of base, which have allowed us to 


\begin{tabular}{ccccccccccccccccc}
$\mu$ & $\alpha_{1}$ & $\alpha_{2}$ & $\alpha_{3}$ & $\alpha_{4}$ & $\alpha_{5}$ & $\alpha_{6}$ & $\alpha_{7}$ & $\alpha_{8}$ & $\alpha_{9}$ & $\alpha_{10}$ & $\alpha_{11}$ & $\alpha_{12}$ & $\alpha_{13}$ & $\alpha_{14}$ & $\alpha_{15}$ & $\alpha_{16}$ \\
\hline 20 & 1 & 0 & 0 & 0 & $\lambda_{1}$ & 1 & $\lambda_{2}$ & 0 & 1 & 1 & 0 & 0 & 0 & 0 & -2 & $-1-3 \lambda_{2}$ \\
21 & 1 & 0 & 0 & 0 & 0 & 1 & $\lambda$ & 0 & 1 & 3 & 0 & 0 & 0 & 0 & -2 & $5-5 \lambda$ \\
22 & 1 & 0 & 0 & 0 & $2 / 3$ & 1 & 0 & 0 & 1 & 3 & 0 & 0 & 0 & 0 & -2 & 5 \\
23 & 1 & 0 & 0 & 0 & $2 / 3$ & 1 & $7 / 9$ & 0 & 1 & 3 & 0 & 0 & 0 & 0 & -2 & $10 / 9$ \\
24 & 0 & 0 & 0 & 0 & 1 & 1 & $\lambda$ & 0 & 1 & $\neq 3$ & 0 & 0 & 0 & 0 & -2 & $-3 \lambda$ \\
25 & 0 & 0 & 0 & 0 & 1 & 1 & 1 & 0 & 1 & 3 & 0 & 0 & 0 & 0 & -2 & -5 \\
26 & 0 & 0 & 0 & 0 & 1 & 1 & 0 & 0 & 1 & 3 & 0 & 0 & 0 & 0 & -2 & 0 \\
27 & 0 & 0 & 0 & 0 & 0 & 1 & 1 & 0 & 1 & 1 & 0 & 0 & 0 & 0 & -2 & -3 \\
28 & 0 & 0 & 0 & 0 & 0 & 1 & 0 & 0 & 1 & 1 & 0 & 0 & 0 & 0 & -2 & 0 \\
29 & 0 & 0 & 0 & 0 & 0 & 1 & 1 & 0 & 1 & 3 & 0 & 0 & 0 & 0 & -2 & -5 \\
30 & 0 & 0 & 0 & 0 & 0 & 1 & 0 & 0 & 1 & 3 & 0 & 0 & 0 & 0 & -2 & 0 \\
31 & 1 & 0 & 0 & 0 & 0 & 1 & $\lambda$ & 0 & 1 & 0 & 0 & 0 & 0 & 0 & -2 & $-4-2 \lambda$ \\
32 & 1 & 0 & 0 & 0 & $2 / 3$ & 1 & 0 & 0 & 1 & 0 & 0 & 0 & 0 & 0 & -2 & -4 \\
33 & 1 & 0 & 0 & 0 & $2 / 3$ & 1 & $-1 / 3$ & 0 & 1 & 0 & 0 & 0 & 0 & 0 & -2 & $-10 / 3$ \\
34 & 0 & 0 & 0 & 0 & 1 & 1 & 1 & 0 & 1 & 0 & 0 & 0 & 0 & 0 & -2 & -2 \\
35 & 0 & 0 & 0 & 0 & 1 & 1 & 0 & 0 & 1 & 0 & 0 & 0 & 0 & 0 & -2 & 0 \\
36 & 0 & 0 & 0 & 0 & 0 & 1 & 1 & 0 & 1 & 0 & 0 & 0 & 0 & 0 & -2 & -2 \\
37 & 0 & 0 & 0 & 0 & 0 & 1 & 0 & 0 & 1 & 0 & 0 & 0 & 0 & 0 & -2 & 0 \\
\hline
\end{tabular}

$\mathrm{Aa}$ Classifcation criterion

Aa Classifcation criterion from previous cases

Aa Additional result from previous cases

$\mathrm{Aa}$ Direct result

FIgURE 3: Case B.1.1: $\alpha_{11}=0, \alpha_{9} \neq 0$, and $\alpha_{6} \neq 0$; classification of the QFLA of dimension 9.

\begin{tabular}{ccccccccccccccccc}
$\mu$ & $\alpha_{1}$ & $\alpha_{2}$ & $\alpha_{3}$ & $\alpha_{4}$ & $\alpha_{5}$ & $\alpha_{6}$ & $\alpha_{7}$ & $\alpha_{8}$ & $\alpha_{9}$ & $\alpha_{10}$ & $\alpha_{11}$ & $\alpha_{12}$ & $\alpha_{13}$ & $\alpha_{14}$ & $\alpha_{15}$ & $\alpha_{16}$ \\
\hline 38 & 1 & $\lambda_{1}$ & $\lambda_{2}$ & 0 & 0 & 0 & 1 & 0 & 1 & 1 & 0 & 0 & 0 & 0 & 0 & 2 \\
39 & 1 & 1 & $\lambda$ & 0 & 0 & 0 & 3 & 0 & 1 & 1 & 0 & 0 & 0 & 0 & 0 & 0 \\
40 & 1 & -2 & 1 & 0 & 0 & 0 & 3 & 0 & 1 & 1 & 0 & 0 & 0 & 0 & 0 & 0 \\
41 & 1 & -2 & 5 & 0 & 0 & 0 & 3 & 0 & 1 & 1 & 0 & 0 & 0 & 0 & 0 & 0 \\
42 & 0 & 1 & 1 & 0 & 0 & 0 & $\lambda$ & 0 & 1 & 1 & 0 & 0 & 0 & 0 & 0 & $-\lambda$ \\
43 & 0 & 1 & 0 & 0 & 0 & 0 & 1 & 0 & 1 & 1 & 0 & 0 & 0 & 0 & 0 & -1 \\
44 & 0 & 1 & 0 & 0 & 0 & 0 & 0 & 0 & 1 & 1 & 0 & 0 & 0 & 0 & 0 & 0 \\
45 & 0 & 0 & 1 & 0 & 0 & 0 & 1 & 0 & 1 & 1 & 0 & 0 & 0 & 0 & 0 & -1 \\
46 & 0 & 0 & 1 & 0 & 0 & 0 & 0 & 0 & 1 & 1 & 0 & 0 & 0 & 0 & 0 & 0 \\
47 & 0 & 0 & 0 & 0 & 0 & 0 & 1 & 0 & 1 & 1 & 0 & 0 & 0 & 0 & 0 & -1 \\
48 & 0 & 0 & 0 & 0 & 0 & 0 & 0 & 0 & 1 & 1 & 0 & 0 & 0 & 0 & 0 & 0 \\
49 & 1 & 0 & 1 & 0 & 0 & 0 & $\lambda$ & 0 & 1 & 0 & 0 & 0 & 0 & 0 & 0 & 0 \\
50 & 1 & 0 & 0 & 0 & 0 & 0 & $\lambda$ & 0 & 1 & 0 & 0 & 0 & 0 & 0 & 0 & 0 \\
51 & 1 & 0 & 1 & $\lambda$ & 0 & 0 & 1 & 0 & 1 & 0 & 0 & 0 & 0 & 0 & 0 & 0 \\
52 & 1 & 0 & 0 & 1 & 0 & 0 & 1 & 0 & 1 & 0 & 0 & 0 & 0 & 0 & 0 & 0 \\
53 & 1 & 0 & 0 & 0 & 0 & 0 & 1 & 0 & 1 & 0 & 0 & 0 & 0 & 0 & 0 & 0 \\
54 & 0 & 1 & 1 & 0 & 0 & 0 & 1 & 0 & 1 & 0 & 0 & 0 & 0 & 0 & 0 & 0 \\
55 & 0 & 1 & 1 & 0 & 0 & 0 & 0 & 0 & 1 & 0 & 0 & 0 & 0 & 0 & 0 & 0 \\
56 & 0 & 1 & 0 & 0 & 0 & 0 & 1 & 0 & 1 & 0 & 0 & 0 & 0 & 0 & 0 & 0 \\
57 & 0 & 1 & 0 & 0 & 0 & 0 & 0 & 0 & 1 & 0 & 0 & 0 & 0 & 0 & 0 & 0 \\
58 & 0 & 0 & 1 & 0 & 0 & 0 & 1 & 0 & 1 & 0 & 0 & 0 & 0 & 0 & 0 & 0 \\
59 & 0 & 0 & 1 & 1 & 0 & 0 & 0 & 0 & 1 & 0 & 0 & 0 & 0 & 0 & 0 & 0 \\
60 & 0 & 0 & 1 & 0 & 0 & 0 & 0 & 0 & 1 & 0 & 0 & 0 & 0 & 0 & 0 & 0 \\
61 & 0 & 0 & 0 & 0 & 0 & 0 & 1 & 0 & 1 & 0 & 0 & 0 & 0 & 0 & 0 & 0 \\
62 & 0 & 0 & 0 & 1 & 0 & 0 & 0 & 0 & 1 & 0 & 0 & 0 & 0 & 0 & 0 & 0 \\
63 & 0 & 0 & 0 & 0 & 0 & 0 & 0 & 0 & 1 & 0 & 0 & 0 & 0 & 0 & 0 & 0 \\
\hline
\end{tabular}

Aa Classification criterion

Classifcation criterion from previous cases

Additional result

Aa Additional result from previous cases

Aa Direct result

FIGURE 4: Case B.1.2: $\alpha_{11}=0 ; \alpha_{9} \neq 0$, and $\alpha_{6}=0$; classification of the QFLA of dimension 9. 


\begin{tabular}{|c|c|c|c|c|c|c|c|c|c|c|c|c|c|c|c|c|}
\hline$\mu$ & $\alpha_{1}$ & $\alpha_{2}$ & $\alpha_{3}$ & $\alpha_{4}$ & $\alpha_{5}$ & $\alpha_{6}$ & $\alpha_{7}$ & $\alpha_{8}$ & $\alpha_{9}$ & $\alpha_{10}$ & $\alpha_{11}$ & $\alpha_{12}$ & $\alpha_{13}$ & $\alpha_{14}$ & $\alpha_{15}$ & $\alpha_{16}$ \\
\hline 64 & 1 & 1 & $\lambda 1$ & 0 & 1 & 0 & 3 & 0 & 0 & 1 & 0 & 0 & 0 & 0 & $\lambda 2 \neq 0,3$ & $\lambda 3$ \\
\hline 65 & 1 & 0 & $\lambda 1$ & 0 & 1 & 0 & 3 & 0 & 0 & 1 & 0 & 0 & 0 & 0 & 3 & $\lambda 2$ \\
\hline 66 & 1 & $\lambda 1-2$ & $\lambda 2$ & 0 & 1 & 0 & 3 & 0 & 0 & 1 & 0 & 0 & 0 & 0 & $\lambda 1 \neq 0,3$ & 0 \\
\hline 67 & 1 & $\lambda-2$ & 0 & 0 & 1 & 0 & 3 & 0 & 0 & 1 & 0 & 0 & 0 & 0 & $\neq 0,3,31 / 8$ & $-5 \lambda$ \\
\hline 68 & 1 & $15 / 8$ & 1 & 0 & 1 & 0 & 3 & 0 & 0 & 1 & 0 & 0 & 0 & 0 & $31 / 8$ & $-155 / 8$ \\
\hline 69 & 1 & $\lambda-2$ & $31-8 \lambda$ & 0 & 1 & 0 & 3 & 0 & 0 & 1 & 0 & 0 & 0 & 0 & $\lambda \ll 0,3$ & $-5 \lambda$ \\
\hline 70 & 1 & 1. & $\lambda$ & 0 & 1 & 0 & 3 & 0 & 0 & 1 & 0 & 0 & 0 & 0 & 3 & 0 \\
\hline 71 & 1 & 1 & 0 & 0 & 1 & 0 & 3 & 0 & 0 & 1 & 0 & 0 & 0 & 0 & 3 & -15 \\
\hline 72 & 1 & 1 & 7 & 0 & 1 & 0 & 3 & 0 & 0 & 1 & 0 & 0 & 0 & 0 & 3 & -15 \\
\hline 73 & 1 & $\lambda_{1}$ & $\lambda 2$ & 0 & 1 & 0 & 3 & 0 & 0 & 1 & 0 & 0 & 0 & 0 & 0 & 1 \\
\hline 74 & 1 & 0 & $\lambda$ & 0 & 1 & 0 & 3 & 0 & 0 & 1 & 0 & 0 & 0 & 0 & 0 & 0 \\
\hline 75 & 1 & -2 & 0 & 0 & 1 & 0 & 3 & 0 & 0 & 1 & 0 & 0 & 0 & 0 & 0 & 0 \\
\hline 76 & 1 & -2 & 31 & 0 & 1 & 0 & 3 & 0 & 0 & 1 & 0 & 0 & 0 & 0 & 0 & 0 \\
\hline 77 & 0 & $\lambda_{1}$ & $\lambda 2$ & 0 & 1 & 0 & 0 & 0 & 0 & 1 & 0 & 0 & 0 & 0 & 1 & 1 \\
\hline 78 & 0 & $\lambda$ & 1 & 0 & 1 & 0 & 0 & 0 & 0 & 1 & 0 & 0 & 0 & 0 & 1 & 0 \\
\hline 79 & 0 & 0 & 0 & 0 & 1 & 0 & 0 & 0 & 0 & 1 & 0 & 0 & 0 & 0 & 1 & 0 \\
\hline 80 & 0 & 1 & 0 & 0 & 1 & 0 & 0 & 0 & 0 & 1 & 0 & 0 & 0 & 0 & 1 & 0 \\
\hline 81 & 0 & 1 & $\lambda$ & 0 & 1 & 0 & 0 & 0 & 0 & 1 & 0 & 0 & 0 & 0 & 0 & 1 \\
\hline 82 & 0 & 0 & 0 & 0 & 1 & 0 & 0 & 0 & 0 & 1 & 0 & 0 & 0 & 0 & 0 & 1 \\
\hline 83 & 0 & 0 & -1 & 0 & 1 & 0 & 0 & 0 & 0 & 1 & 0 & 0 & 0 & 0 & 0 & 1 \\
\hline 84 & 0 & 1 & 1 & 0 & 1 & 0 & 0 & 0 & 0 & 1 & 0 & 0 & 0 & 0 & 0 & 0 \\
\hline 85 & 0 & 1 & 0 & 0 & 1 & 0 & 0 & 0 & 0 & 1 & 0 & 0 & 0 & 0 & 0 & 0 \\
\hline 86 & 0 & 0 & 1 & 0 & 1 & 0 & 0 & 0 & 0 & 1 & 0 & 0 & 0 & 0 & 0 & 0 \\
\hline 87 & 0 & 0 & 0 & 0 & 1 & 0 & 0 & 0 & 0 & 1 & 0 & 0 & 0 & 0 & 0 & 0 \\
\hline 88 & 1 & 0 & 0 & 0 & 1 & 0 & $\lambda 1 \neq 1 / 2$ & $\lambda 2$ & 0 & 0 & 0 & 0 & 0 & 0 & $\lambda 3 \neq 2$ & 1 \\
\hline 89 & 1 & 0 & 0 & 0 & 1 & 0 & $\lambda_{1} \neq 1 / 2$ & 1 & 0 & 0 & 0 & 0 & 0 & 0 & $\lambda 2 \neq 2$ & 0 \\
\hline 90 & 1 & 1 & 0 & 0 & 1 & 0 & $\lambda 1 \neq 0$ & $\lambda 2$ & 0 & 0 & 0 & 0 & 0 & 0 & 2 & 0 \\
\hline 91 & 1 & 1 & 0 & 0 & 1 & 0 & 0 & 0 & 0 & 0 & 0 & 0 & 0 & 0 & 2 & $\lambda$ \\
\hline 92 & 1 & 0 & 0 & 0 & 1 & 0 & $\lambda \neq 0$ & 1 & 0 & 0 & 0 & 0 & 0 & 0 & 2 & 0 \\
\hline 93 & 1 & 0 & 0 & 0 & 1 & 0 & $\lambda \neq 0$ & 0 & 0 & 0 & 0 & 0 & 0 & 0 & 2 & 0 \\
\hline 94 & 1 & 0 & 0 & 0 & 1 & 0 & 0 & 0 & 0 & 0 & 0 & 0 & 0 & 0 & 2 & 1 \\
\hline 95 & 1 & 0 & 0 & 0 & 1 & 0 & 0 & 0 & 0 & 0 & 0 & 0 & 0 & 0 & 2 & 0 \\
\hline 96 & 0 & 1 & 0 & 0 & 1 & 0 & 1 & 0 & 0 & 0 & 0 & 0 & 0 & 0 & $\lambda_{1}$ & $\lambda 2$ \\
\hline 97 & 0 & 0 & 0 & 0 & 1 & 0 & 1 & 0 & 0 & 0 & 0 & 0 & 0 & 0 & $\lambda$ & 1 \\
\hline 98 & 0 & 0 & 0 & 0 & 1 & 0 & 1 & 0 & 0 & 0 & 0 & 0 & 0 & 0 & $\lambda$ & 0 \\
\hline 99 & 1 & $\lambda_{1}$ & $\lambda 2$ & 0 & 1 & 0 & 1 & 0 & 0 & 0 & 0 & 0 & 0 & 0 & $\lambda 3$ & 1 \\
\hline 100 & 1 & $\lambda 1$ & 1 & 0 & 1 & 0 & . & 0 & 0 & 0 & 0 & 0 & 0 & 0 & $\lambda 2$ & 0 \\
\hline 101 & 1 & 1 & 0 & 0 & 1 & 0 & 1 & 0 & 0 & 0 & 0 & 0 & 0 & 0 & $\lambda$ & 0 \\
\hline 102 & 1 & 0 & 0 & 0 & 1 & 0 & 1 & 0 & 0 & 0 & 0 & 0 & 0 & 0 & $\lambda$ & 0 \\
\hline 103 & 1 & $\lambda 1$ & 0 & 0 & 1 & 0 & $1 / 2$ & (1) & 0 & 0 & 0 & 0 & 0 & 0 & $\lambda 2$ & 1 \\
\hline 104 & 1 & 1 & 0 & 0 & 1 & 0 & $1 / 2$ & 0 & 0 & 0 & 0 & 0 & 0 & 0 & $\lambda$ & 0 \\
\hline 105 & 1 & 0 & 0 & 0 & 1 & 0 & $1 / 2$ & 0 & 0 & 0 & 0 & 0 & 0 & 0 & $\lambda$ & 0 \\
\hline 106 & 0 & $\lambda_{1}$ & 0 & 0 & 1 & 0 & 0 & 1 & 0 & 0 & 0 & 0 & 0 & 0 & $\lambda 2$ & 1 \\
\hline 107 & 0 & 0 & $\lambda$ & 0 & 1 & 0 & 0 & 1 & 0 & 0 & 0 & 0 & 0 & 0 & 1 & 0 \\
\hline 108 & 0 & $\lambda$ & 1 & 0 & 1 & 0 & 0 & 1 & 0 & 0 & 0 & 0 & 0 & 0 & 0 & 0 \\
\hline 109 & 0 & $\lambda$ & 0 & 0 & 1 & 0 & 0 & 1 & 0 & 0 & 0 & 0 & 0 & 0 & 0 & 0 \\
\hline 110 & 0 & 0 & $\lambda$ & 0 & 1 & 0 & 0 & 0 & 0 & 0 & 0 & 0 & 0 & 0 & 1 & 1 \\
\hline 111 & 0 & 0 & 1 & 0 & 1 & 0 & 0 & 0 & 0 & 0 & 0 & 0 & 0 & 0 & 1 & 0 \\
\hline 112 & 0 & 0 & 0 & 0 & 1 & 0 & 0 & 0 & 0 & 0 & 0 & 0 & 0 & 0 & 1 & 0 \\
\hline 113 & 0 & 1 & 0 & 0 & 1 & 0 & 0 & 0 & 0 & 0 & 0 & 0 & 0 & 0 & 0 & 1 \\
\hline 114 & 0 & 1 & 1 & 0 & 1 & 0 & 0 & 0 & 0 & 0 & 0 & 0 & 0 & 0 & 0 & 0 \\
\hline 115 & 0 & 1 & 0 & 0 & 1 & 0 & 0 & 0 & 0 & 0 & 0 & 0 & 0 & 0 & 0 & 0 \\
\hline 116 & 0 & 0 & 0 & 0 & 1 & 0 & 0 & 0 & 0 & 0 & 0 & 0 & 0 & 0 & 0 & 1 \\
\hline 117 & 0 & 0 & 1 & 0 & 1 & 0 & 0 & 0 & 0 & 0 & 0 & 0 & 0 & 0 & 0 & 0 \\
\hline 118 & 0 & 0 & 0 & 0 & 1 & 0 & 0 & 0 & 0 & 0 & 0 & 0 & 0 & 0 & 0 & 0 \\
\hline
\end{tabular}

Aa Classification criterion

4a Classifcation criterion from previous cases

Additional result

$\mathrm{Aa}$ Additional result from previous cases

Aa Direct result

FIgURE 5: Case B.2.1: $\alpha_{11}=0, \alpha_{9}=0, \alpha_{5} \neq 0$; classification of the QFLA of dimension 9. 


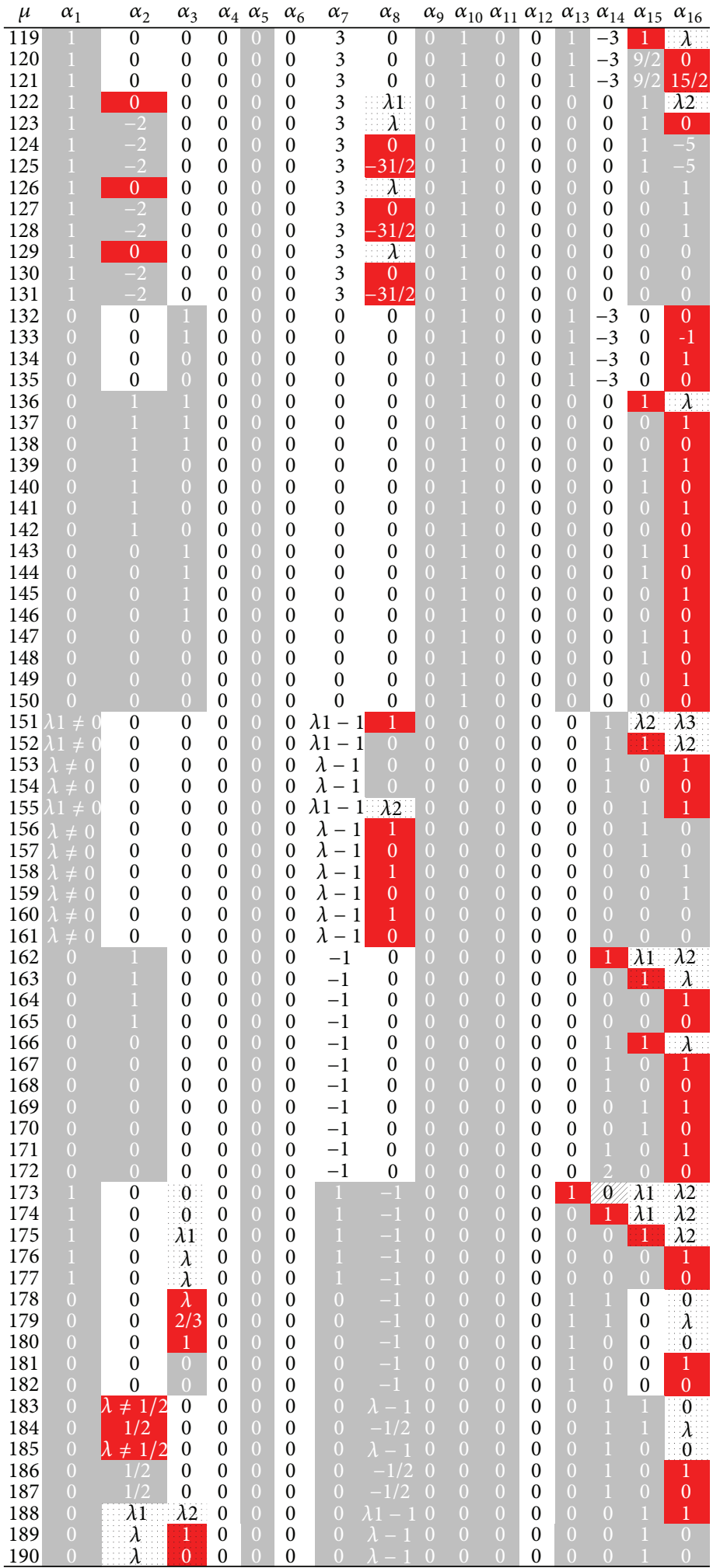

Aa Classification criterion

Classifcation criterion from previous cases

Additional result

$\mathrm{Aa}$ Additional result from previous cases

Aa Direct result

FIgURE 6: Case B.2.2. First part: $\alpha_{11}=0, \alpha_{9}=0$, and $\alpha_{5}=0$. Classification of the QFLA of dimension 9. 


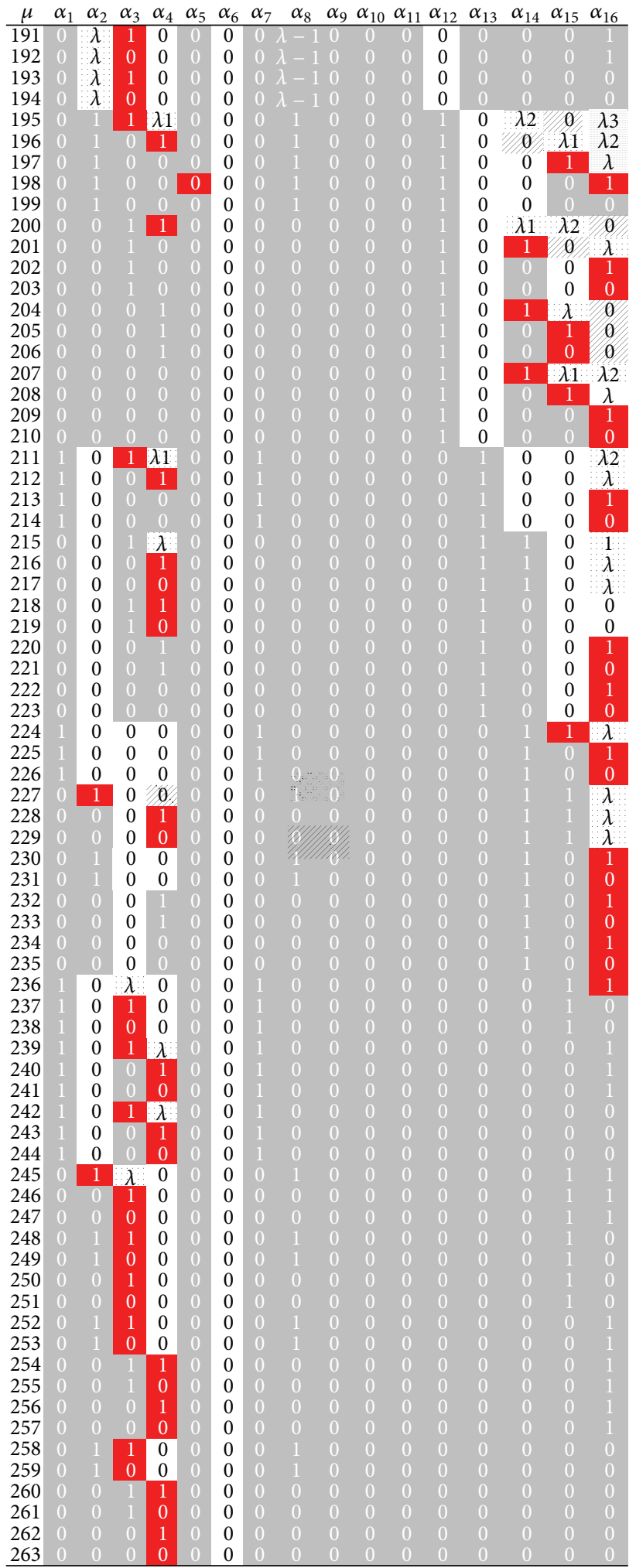

Aa Classification criterion

Aa Classifcation criterion from previous cases

Additional result

Aa Additional result from previous cases

$\mathrm{Aa}$ Direct result

FIgURE 7: Case B.2.2. Second part: $\alpha_{11}=0, \alpha_{9}=0$, and $\alpha_{5}=0$. Classification of the QFLA of dimension 9. 
eliminate some parameters in the laws involved. In any case, the massive application of changes of base and characteristic vector has allowed us to obtain the complete classification in 263 subfamilies of the QFLA laws of dimension 9.

The 263 families have been represented in the paper, consisting of 157 simple algebras, 77 families depending on 1 parameter, 24 families depending on 2 parameters, and 5 families depending on 3 parameters. The classification is complete since any couple of the obtained 263 families is nonisomorphic and any quasifiliform Lie algebra of dimension 9 is isomorphic to one of them. The nonisomorphism of the 263 Lie algebra families has been proved in the 10 propositions of the paper, and the completeness of the classification is proved by the "exhaustive" analysis of all the possible cases, depending on the combination of the values of the 16 parameters $(a 1 \cdots a 16)$.

\section{Conflict of Interests}

The authors declare that there is no conflict of interests regarding the publication of this paper.

\section{Acknowledgment}

The authors appreciate the aid of José Ramón Gómez Martn, Professor of the University of Seville, advisor of the Ph.D. thesis of one of the authors, which constitutes the basis of these works.

\section{References}

[1] H. Georgi, Lie Algebras in Particle Physics: From Isospin to Unified Theories (Frontiers in Physics), Westview Press, Boulder, Colo, USA, 1999.

[2] R. Gilmore, Lie Groups, Lie Algebras, and Some of Their Applications, Dover, New York, NY, USA, 2005.

[3] W. A. De Graaf, "Constructing algebraic groups from their Lie algebras," Journal of Symbolic Computation, vol. 44, no. 9, pp. 1223-1233, 2009.

[4] R. J. Moitsheki and M. D. Mhlongo, "Classical Lie point symmetry analysis of a steady nonlinear one-dimensional fin problem," Journal of Applied Mathematics, vol. 2012, Article ID 671548, 13 pages, 2012.

[5] K. S. Govinder, "Symbolic implementation of preliminary group classiffication for ordinary differential equations," Journal of Applied Mathematics, vol. 2013, Article ID 976271, 6 pages, 2013.

[6] M. Goze and Y. Khakimdjanov, Nilpotent Lie Algebras, Kluwer Academic, New York, NY, USA, 1996.

[7] D. Burde, B. Eick, and W. de Graaf, "Computing faithful representations for nilpotent Lie algebras," Journal of Algebra, vol. 322, no. 3, pp. 602-612, 2009.

[8] J. C. Benjumea, J. Núñez, and F. Tenorio, "Computing the law of a family of solvable Lie algebras," International Journal of Algebra and Computation, vol. 19, no. 3, pp. 337-345, 2009.

[9] C. Schneider, "A computer-based approach to the classification of nilpotent Lie algebras," Experimental Mathematics, vol. 14, no. 2, pp. 153-160, 2005.

[10] J. M. Ancochéa-Bermúdez and M. Goze, "Sur la classification des algèbres de Lie nilpotentes de dimension 7," Comptes Rendus des Séances de l'Académie des Sciences. Série I. Mathématique, vol. 302, no. 17, pp. 611-613, 1986.

[11] J. M. Ancochéa-Bermúdez and M. Goze, "Classification des algèbres de Lie nilpotentes complexes de dimension 7," Archiv der Mathematik, vol. 52, no. 2, pp. 175-185, 1989.

[12] J. M. Ancochéa-Bermúdez and M. Goze, "Classification des algèbres de Lie filiformes de dimension 8," Archiv der Mathematik, vol. 50, no. 6, pp. 511-525, 1988.

[13] J. R. Gomez and F. J. Echarte, "Classification of complex filiform nilpotent Lie algebras of dimension 9," Rendiconti del Seminario della Facoltà di Scienze dell'Università di Cagliari, vol. 61, no. 1, pp. 21-29, 1991.

[14] F. J. Castro, J. R. Gomez, A. Jiménez-Merchan, N. Nunez, and G. Valeiras, "Determination of law families of fliliform Lie algebres," in Proceedings of the Workshop of Matricial Analysis and Applications, Vitoria, Spain, 1995.

[15] J. R. Gomez and A. Jiménez-Merchan, "Naturally graded quasifiliform Lie algebras," Journal of Algebra, vol. 256, no. 1, pp. 211228, 2002.

[16] L. García-Vergnolle, "Sur les algèbres de Lie quasi-filiformes admettant un tore de dérivations," Manuscripta Mathematica, vol. 124, no. 4, pp. 489-505, 2007.

[17] M. Vergne, Varieté des algèbres de Lie nilpotentes [Ph.D. thesis], Kluwer Academic, Paris, France, 1966.

[18] M. Vergne, "Cohomologie des algèbres de Lie nilpotentes. Application à l'étude de la variété des algèbres de Lie nilpotentes," Bulletin de la Société Mathématique de France, vol. 98, pp. 81-116, 1970.

[19] F. Pérez, Clasificacion de las algebras de lie cuasifiliformes de dimension 9 [Ph.D. thesis], University of Sevilla, Sevilla, Spain, 2007.

[20] G. G. A. Bäuerle and E. A. de Kerf, Lie Algebras Part 1, Studies in Mathematical Physics 1, Elsevier, New York, NY, USA, 1990.

[21] J. C. Benjumea, D. Fernandez, M. C. Márquez, J. Nuñez, and J. A. Vilches, Matemáticas Avanzadas y Estadística para Ciencias e Ingenierías, Secretariado de Publicaciones de la Universidad de Sevilla, Sevilla, Spain, 2006.

[22] J. R. Sendra, S. Perez-Diaz, J. Sendra, and C. Villarino, Introducción a la Computación Simbólica y Facilidades Maple, Addlink Media, 2009.

[23] M. Goze, "Perturbations of Lie algebra structures," in Deformation Theory of Algebras and Structures and Applications, M. Hazewinkel and M. Gerstenhaber, Eds., pp. 265-355, Kluwer, New York, NY, USA, 1988. 


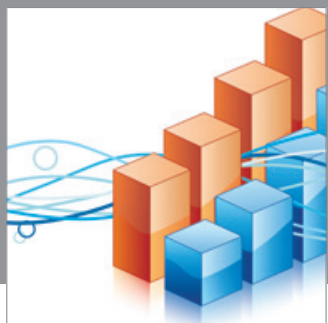

Advances in

Operations Research

mansans

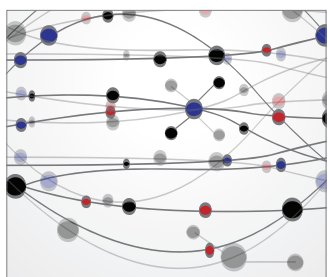

The Scientific World Journal
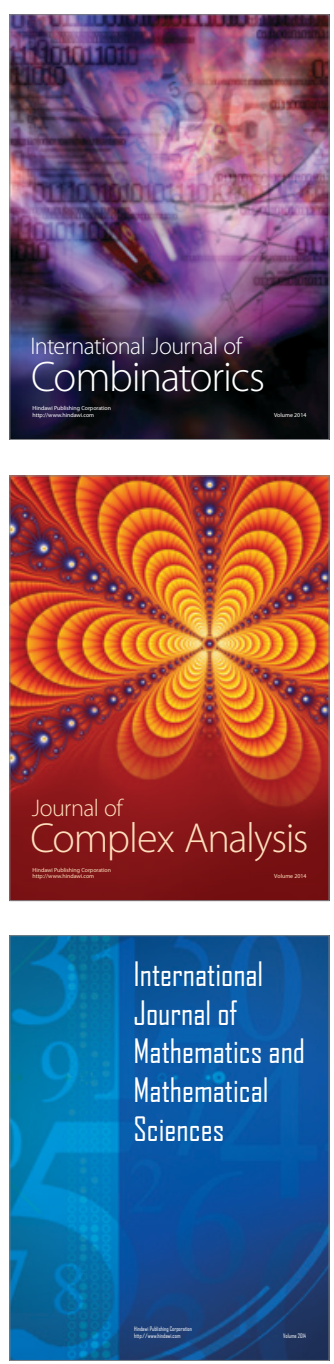
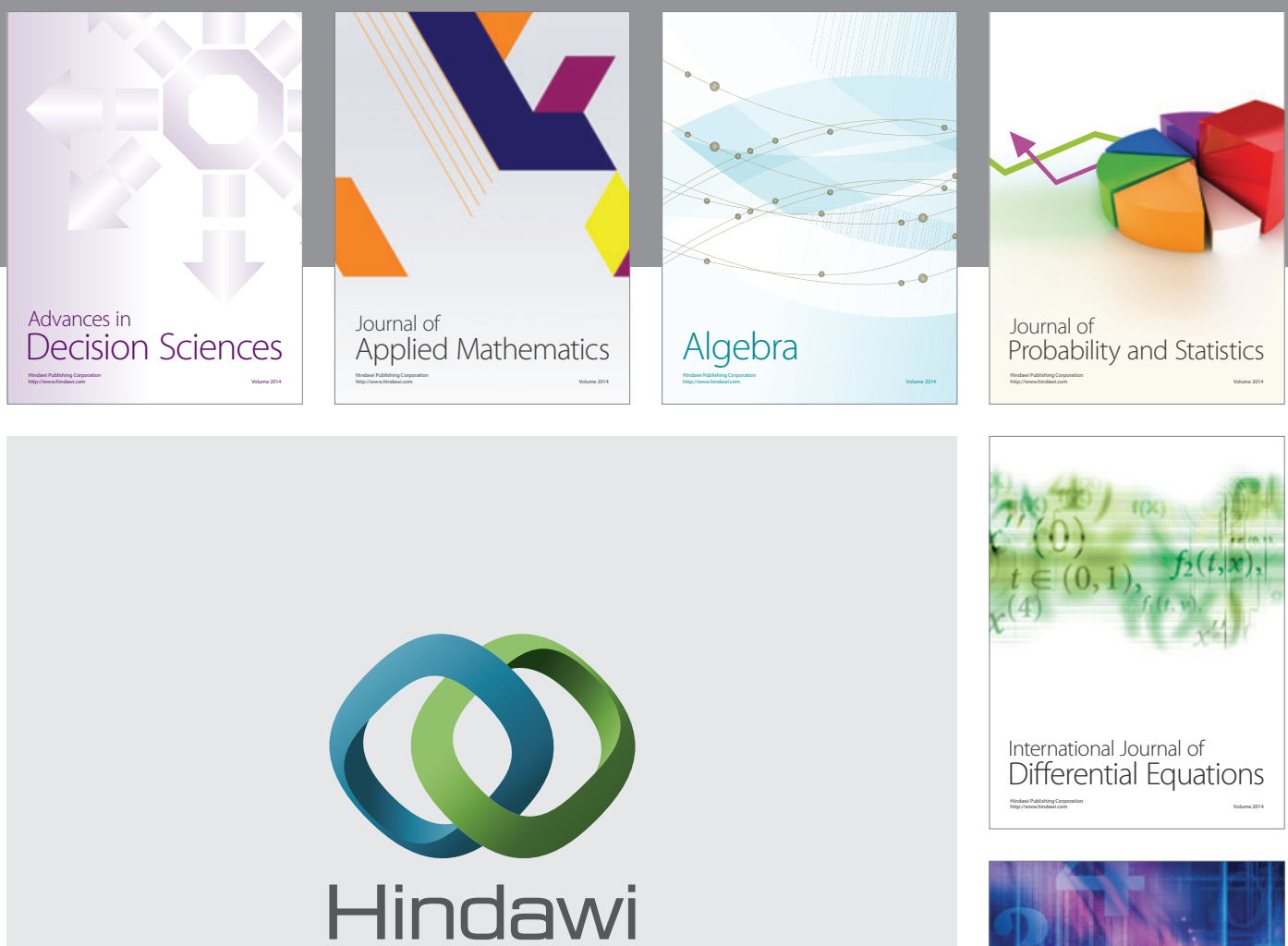

Submit your manuscripts at http://www.hindawi.com
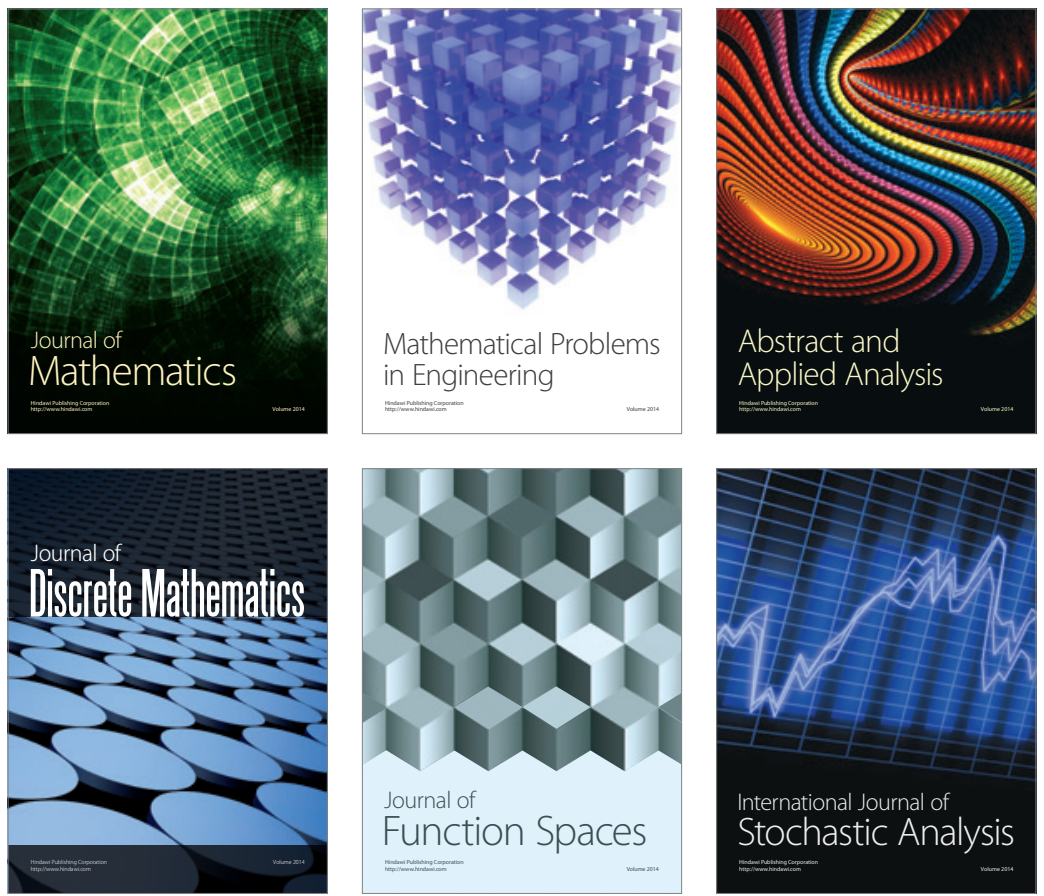

Journal of

Function Spaces

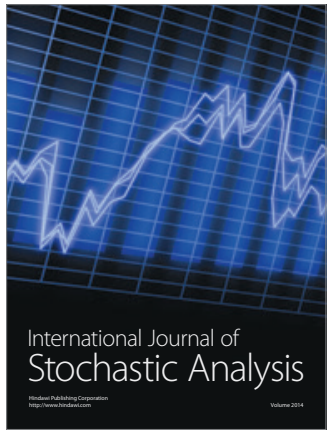

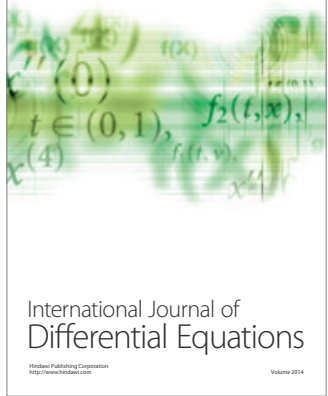
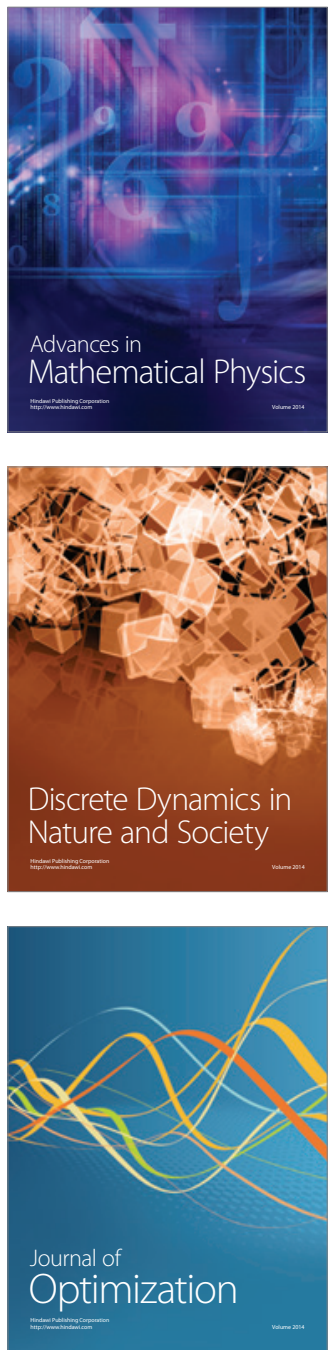